\title{
Pareto Frontier of the Arm Energy Ripple and the Conduction Losses of a Modular Multilevel Converter
}

\author{
Mario Lopez ${ }^{1, *}$, Hendrik Fehr ${ }^{2, * \mathbb{D}}$, Marcelo A. Perez ${ }^{1}$ (D) and Albrecht Gensior ${ }^{2}$ (D) \\ 1 Electronics Engineering Department, Universidad Técnica Federico Santa María, Valparaíso 2390123, Chile; \\ marcelo.perez@usm.cl \\ 2 Professur Leistungselektronik, Elektrotechnisches Institut, Technische Universität Dresden, \\ 01062 Dresden, Germany; albrecht.gensior@tu-dresden.de \\ * Correspondence: mario.lopez@alumnos.usm.cl (M.L.); hendrik.fehr@tu-dresden.de (H.F.)
}

\begin{abstract}
Modular multilevel converters can achieve high power quality and voltage ratings, becoming a good alternative for high-voltage direct-current applications. However, the circulating current and capacitor voltage ripple remain significant drawbacks, mainly due to the impact on efficiency and power density. In this work, a stationary operation of a modular multilevel converter is optimized by calculating the Pareto frontier of the arm energy ripple and the conduction losses for different operating conditions. From the set of optimal solutions, the best trade-off between energy ripple and power losses can be chosen depending on the application requirements. Therefore, an optimal operating point regarding capacitor voltage ripple and circulating current was found, optimizing efficiency and allowing a reduction of capacitance, hence size and cost. The theoretical analysis and results are validated experimentally on a prototype test-bench.
\end{abstract}

Keywords: multilevel converters; power converter control; Pareto frontier

Citation: Lopez, M.; Fehr, H.; Perez, M.A.; Gensior, A. Pareto Frontier of the Arm Energy Ripple and the Conduction Losses of a Modular Multilevel Converter. Energies 2021, 14, 392. https://doi.org/10.3390/ en14020392

Received: 12 November 2020 Accepted: 25 December 2020 Published: 12 January 2021

Publisher's Note: MDPI stays neutral with regard to jurisdictional clai$\mathrm{ms}$ in published maps and institutional affiliations.

Copyright: (C) 2021 by the authors. Licensee MDPI, Basel, Switzerland. This article is an open access article distributed under the terms and conditions of the Creative Commons Attribution (CC BY) license (https:// creativecommons.org/licenses/by/ $4.0 /)$.

\section{Introduction}

The Modular Multilevel Converter (MMC) provides several advantages such as high voltage ratings, high power quality, modularity, and transformerless operation, making it attractive for medium- and high-voltage power conversion applications [1-4]. The MMC is already employed in high-voltage DC transmission [5-8], photovoltaic power plants [9,10], and medium-voltage motor applications [11].

A standard configuration of the MMC consists of six arms arranged as a three-phase bridge, as shown in Figure 1a. Each arm is a series connection of half-bridge [12] or full bridge [13] cells with a floating capacitor and an arm inductor to suppress high-frequency currents $[14,15]$. Despite all the mentioned MMC advantages, this converter also has several drawbacks, such as the existence of a circulating current among arms [16] and the voltage ripple in the floating capacitors [17]. The former can increase the losses in the arms and reduce the overall efficiency and the latter requires a large cell capacitance to minimize the impact of the voltage ripple in the other variables.

The minimization of the capacitor voltage ripple has become a relevant topic due to the reduction of cell capacitance, hence the size and cost of the converter. There are only two degrees of freedom to reduce the voltage ripple, namely the circulating current and the common-mode voltage. The other controlled variables of the converter, such as the $\mathrm{AC}$ and DC currents, are fixed by the application requirements. Several control schemes to reduce the capacitor voltage ripple have been proposed using only the circulating current $[18,19]$ or using a combination of circulating current and common-mode voltage $[20,21]$. The use of the common-mode voltage can achieve further reduction of the voltage ripple, but it also increases the arm voltage, reducing the available modulation index required for control purposes [22]. 


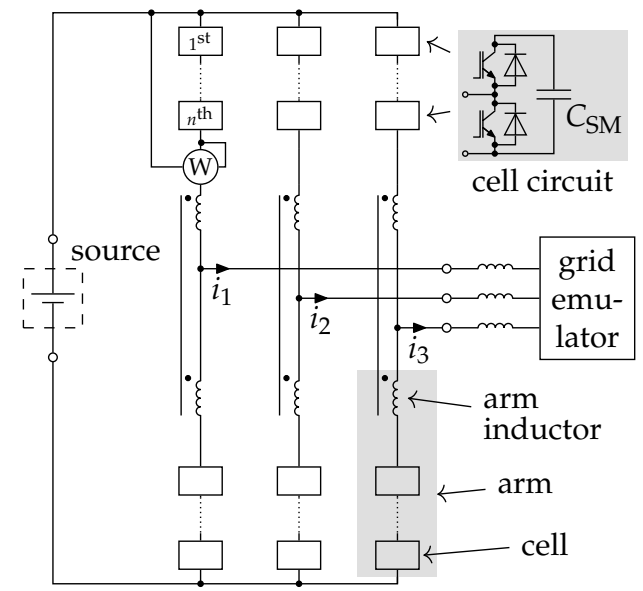

(a) circuit diagram

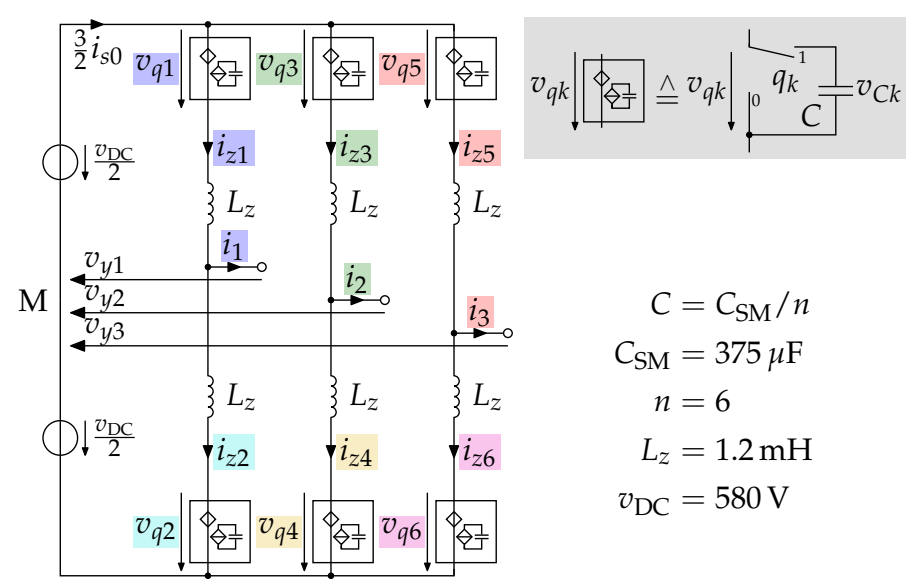

(b) continuous model using equivalent cells

Figure 1. Three phase MMC: (a) circuit, and (b) continuous model. The cells of each arm are represented by equivalent cells and their duty cycles $q_{k} \in[0,1], k=1, \ldots, 6$ are used as control inputs.

Several control methods to achieve the capacitor voltage have been proposed based either on offline, online or analytic algorithms. Offline algorithms are based on a numerical optimization performed offline storing the feasible operating points in a table $[19,23]$, and online algorithms are based on a numerical optimization performed while the converter is operating $[24,25]$, and analytic methods minimize the capacitor voltage, or energy ripple, using a model based optimization, generating the required references for circulating current and common-mode voltage [26-28].

This paper focuses on the analysis of the trade-off between the energy ripple reduction and the conduction losses generated by using the circulating current in the MMCs. A method to numerically solve the multi-objective optimization problem is also proposed and validated experimentally. Therefore, the best combination of both variables can be chosen to fulfill the application requirements.

The paper is organized as follows: An MMC energy model, the operating conditions, the capacitor design, and the model of the conduction losses are introduced in the next section. Section 3 is dedicated to the Pareto frontier calculation while Section 4 reports on its validation using a low-voltage test-bench. Conclusions are given in the end.

\section{Modeling}

In this paper, an MMC with half-bridge cells, which is the most common configuration in HVDC applications, is considered. To simplify the analysis, the series connected cells of each arm are represented by an equivalent cell $[6,29]$ as shown in Figure $1 b$.

\subsection{Arm Energy Dynamics}

The model of the converter is based on the stored energy instead of voltage, in order to simplify the analysis obtaining a direct result for voltages and currents. The series connected cells of each arm are modeled by one equivalent cell, where the stored energy is defined as

$$
e_{z k}=\frac{1}{2} C v_{C k}^{2}+\frac{1}{2} L_{z} i_{z k}^{2}
$$

for each arm $k=1, \ldots, 6$. The previous model considers the equivalent cell capacitance $C$ and the arm inductors self inductance $L_{z}$. The mutual inductance which appears in the case of coupled arm inductors is neglected for the sake of simplification. 
The MMC modeling relies on the usual summation and subtraction of arm variables and their transformation into $0 \alpha \beta$-components as given in Table 1 . For efficient notation, the zero sequence is obtained via the row

$$
g_{0}=\frac{1}{3}(1,1,1)
$$

while a complex notation is obtained for the $\alpha$ - and $\beta$-components by application of the row

$$
\underline{g}_{\alpha \beta}=\frac{1}{3}\left(2, \underline{a}, \underline{a}^{*}\right), \quad \text { with the complex phasor } \quad \underline{a}=-1+j \sqrt{3} .
$$

In (3) and in the following, the notation $\underline{a}^{*}$ indicates the complex conjugate of $\underline{a}$. Table 2 contains inverse transformations, in which $\underline{g}_{\alpha \beta}^{*}=\frac{1}{3}\left(2, \underline{a}^{*}, \underline{a}\right)^{\mathrm{T}}$.

Table 1. Definitions of the transformed arm energies and MMC currents using the row $g_{0}$ defined in (2) and the complex row $\underline{g}_{\alpha \beta}$ given in (3).

\begin{tabular}{rlrl}
\hline \multicolumn{1}{c}{ Definition } & Description \\
\hline$e_{s 0}$ & $=2 g_{0}\left[\left(e_{z 1}, e_{z 3}, e_{z 5}\right)^{\mathrm{T}}+\left(e_{z 2}, e_{z 4}, e_{z 6}\right)^{\mathrm{T}}\right]$ & & twice the total stored energy \\
$e_{d 0}$ & $=2 g_{0}\left[\left(e_{z 1}, e_{z 3}, e_{z 5}\right)^{\mathrm{T}}-\left(e_{z 2}, e_{z 4}, e_{z 6}\right)^{\mathrm{T}}\right]$ & & twice the vertical difference \\
$\underline{e}_{s}$ & $=2 \underline{g}_{\alpha \beta}\left[\left(e_{z 1}, e_{z 3}, e_{z 5}\right)^{\mathrm{T}}+\left(e_{z 2}, e_{z 4}, e_{z 6}\right)^{\mathrm{T}}\right]$ & & complex energy sum in $\alpha \beta$-frame \\
$\underline{e}_{d}$ & $=2 \underline{g}_{\alpha \beta}\left[\left(e_{z 1}, e_{z 3}, e_{z 5}\right)^{\mathrm{T}}-\left(e_{z 2}, e_{z 4}, e_{z 6}\right)^{\mathrm{T}}\right]$ & & complex energy difference in $\alpha \beta$-frame \\
\hline$i_{s 0}$ & $=g_{0}\left[\left(i_{z 1}, i_{z 3}, i_{z 5}\right)^{\mathrm{T}}+\left(i_{z 2}, i_{z 4}, i_{z 6}\right)^{\mathrm{T}}\right]$ & & two thirds of the DC current \\
$\underline{i}_{s}$ & $=\underline{g}_{\alpha \beta}\left[\left(i_{z 1}, i_{z 3}, i_{z 5}\right)^{\mathrm{T}}+\left(i_{z 2}, i_{z 4}, i_{z 6}\right)^{\mathrm{T}}\right]$ & & circulating current in complex $\alpha \beta$-frame \\
0 & $=g_{0}\left[\left(i_{z 1}, i_{z 3}, i_{z 5}\right)^{\mathrm{T}}-\left(i_{z 2}, i_{z 4}, i_{z 6}\right)^{\mathrm{T}}\right]$ & & Kirchhoff's current rule for the AC star point \\
$\underline{i}$ & $=\underline{g}_{\alpha \beta}\left[\left(i_{z 1}, i_{z 3}, i_{z 5}\right)^{\mathrm{T}}-\left(i_{z 2}, i_{z 4}, i_{z 6}\right)^{\mathrm{T}}\right]$ & & output current in complex $\alpha \beta$-frame \\
\hline$v_{x 0}$ & $=v_{\mathrm{DC}}-g_{0}\left[\left(v_{q 1}, v_{q 3}, v_{q 5}\right)^{\mathrm{T}}+\left(v_{q 2}, v_{q 4}, v_{q 6}\right)^{\mathrm{T}}\right]$ & & DC current driving voltage \\
$\underline{v}_{x}$ & $=-\underline{g}_{\alpha \beta}\left[\left(v_{q 1}, v_{q 3}, v_{q 5}\right)^{\mathrm{T}}+\left(v_{q 2}, v_{q 4}, v_{q 6}\right)^{\mathrm{T}}\right]$ & & circulating current driving voltage \\
$v_{y 0}$ & $=g_{0}\left(v_{y 1}, v_{y 2}, v_{y 3}\right)^{\mathrm{T}}$ & & common-mode voltage \\
& $=-\frac{1}{2} g_{0}\left[\left(v_{q 1}, v_{q 3}, v_{q 5}\right)^{\mathrm{T}}-\left(v_{q 2}, v_{q 4}, v_{q 6}\right)^{\mathrm{T}}\right]$ & \\
$\underline{v}_{y}$ & $=\underline{g}_{\alpha \beta}\left(v_{y 1}, v_{y 2}, v_{y 3}\right)^{\mathrm{T}}$ & & output voltage in complex $\alpha \beta$-frame \\
& $=-\frac{1}{2} \underline{g}_{\alpha \beta}\left[\left(v_{q 1}, v_{q 3}, v_{q 5}\right)^{\mathrm{T}}-\left(v_{q 2}, v_{q 4}, v_{q 6}\right)^{\mathrm{T}}\right]$ & \\
\hline
\end{tabular}

Table 2. Calculation of MMC arm energies and currents from the transformed variables in Table 1, in which $\underline{g}_{\alpha \beta}^{*}=\frac{1}{3}\left(2, \underline{a}^{*}, \underline{a}\right)^{\mathrm{T}}$.

\begin{tabular}{cl}
\hline Definition & Description \\
\hline$\left(e_{z 1}, e_{z 3}, e_{z 5}\right)^{\mathrm{T}}=\frac{3}{4} g_{0}^{\mathrm{T}}\left(e_{s 0}+e_{d 0}\right)+\frac{3}{8} \operatorname{Re}\left(\underline{g}_{\alpha \beta}^{*}\left(\underline{e}_{s}+\underline{e}_{d}\right)\right)$ & upper arm energies \\
$\left(e_{z 2}, e_{z 4}, e_{z 6}\right)^{\mathrm{T}}=\frac{3}{4} g_{0}^{\mathrm{T}}\left(e_{s 0}-e_{d 0}\right)+\frac{3}{8} \operatorname{Re}\left(\underline{g}_{\alpha \beta}^{*}\left(\underline{e}_{s}-\underline{e}_{d}\right)\right)$ & lower arm energies \\
\hline$\left(i_{z 1}, i_{z 3}, i_{z 5}\right)^{\mathrm{T}}=\frac{3}{2} g_{0}^{\mathrm{T}} i_{s 0}+\frac{3}{4} \operatorname{Re}\left(\underline{g}_{\alpha \beta}^{*}\left(\underline{i}_{s}+\underline{i}\right)\right)$ & upper arm currents \\
$\left(i_{z 2}, i_{z 4}, i_{z 6}\right)^{\mathrm{T}}=\frac{3}{2} g_{0}^{\mathrm{T}} i_{s 0}+\frac{3}{4} \operatorname{Re}\left(\underline{g}_{\alpha \beta}^{*}\left(\underline{i}_{s}-\underline{i}\right)\right)$ & lower arm currents \\
\hline
\end{tabular}

The MMC model is obtained after inserting the equivalent cell capacitor voltage dynamics

$$
C \frac{\mathrm{d}}{\mathrm{d} t} v_{C k}=\frac{v_{q k}}{v_{C k}} i_{z k}, \quad k=1, \ldots, 6
$$


and the arm current dynamics

$$
L_{z} \frac{\mathrm{d}}{\mathrm{d} t} i_{z k}=v_{q k}+(-1)^{k} v_{y p}-\frac{v_{\mathrm{DC}}}{2}, \quad p=\left\lceil\frac{k}{2}\right\rceil, \quad k=1, \ldots, 6
$$

into the derivation of the arm energy definition (1). Application of the relations from Tables 1 and 2 results in the transformed arm energy dynamics [30]

$$
\begin{aligned}
& \dot{e}_{S 0}=v_{\mathrm{DC}} i_{s 0}-\operatorname{Re}\left(\underline{i} \underline{v}_{y}^{*}\right) \\
& \text { twice the total stored energy } \\
& \dot{e}_{d 0}=-2 v_{y 0} i_{s 0}-\operatorname{Re}\left(\underline{i}_{s}^{*} \underline{v}_{y}\right) \\
& \underline{\dot{e}}_{s}=v_{\mathrm{DC}} \underline{i}_{s}-\underline{v}_{y}^{*} \underline{i}^{*}-2 \underline{i} v_{y 0} \\
& \underline{\dot{e}}_{d}=v_{\mathrm{DC} \underline{i}}-\underline{i}_{s}^{*} \underline{v}_{y}^{*}-2 \underline{i}_{s} v_{y 0}-2 i_{s 0} \underline{v}_{y} . \\
& \text { twice the vertical energy difference } \\
& \text { complex energy sum in } \alpha \beta \text {-frame } \\
& \text { complex energy difference in } \alpha \beta \text {-frame }
\end{aligned}
$$

The real variables $e_{s 0}$ and $e_{d 0}$ represent the stored energy and the vertical energy difference between all upper and all lower arms, respectively. The complex energy sum $\underline{e}_{S}$ and energy difference $\underline{e}_{d}$ together with the vertical difference $e_{d 0}$ represent the alternating part of the arm energies in stationary operation. The circulating current is denoted by $\underline{i}_{s}$. The output current, the output voltage, and the common-mode voltage are denoted by $i, \underline{v}_{y}$, and $v_{y 0}$, respectively.

In the context of the present paper, stationary operating regimes are considered implying all system variables to be periodical in time with the same period. As a consequence, the trajectories can be written as Fourier series. Assuming a stationary symmetric operation on the AC side, constant DC current and voltage on the DC side, and a 3rd harmonic for the common-mode voltage, all variables except for $\underline{i}_{s}$ are fixed by the AC and DC terminal voltages and currents. Thus, it can be written

$$
\begin{aligned}
\underline{v}_{y} & =\hat{V}_{y} \mathrm{e}^{j \theta} & \\
\underline{i} & =\underline{\mathrm{e}}^{\mathrm{j} \theta} & \\
v_{y 0} & =\operatorname{Re}\left(\underline{V}_{y 0}^{[3]} \mathrm{e}^{j 3 \theta}\right) & \underline{V}_{y 0}^{[3]}=-\frac{1}{6} \hat{V}_{y} \\
v_{\mathrm{DC}} & =V_{\mathrm{DC}} & \\
i_{s 0} & =I_{s 0} & I_{s 0}=\operatorname{Re}\left(\underline{I}^{*} \hat{V}_{y}\right) / V_{\mathrm{DC}}
\end{aligned}
$$

with the angle $\theta=\omega t$, the real constants $\omega, I_{s 0}, V_{\mathrm{DC}}, \hat{V}_{y}$ and the complex constants $\underline{V}_{y 0}^{[3]}, \underline{I}$, and $\underline{I}_{s}^{[h]}, h \in \mathbb{Z}$. The first two equations, i.e., (10) and (11), result from assuming a symmetric operation on the AC side with a fundamental angular frequency $\omega$, while (12) implements a 3rd harmonic in the common-mode voltage for modulation region extension. The DC current $I_{s 0}$, as given by (14), ensures that the stored energy $e_{s 0}$ stays constant. While all the other system variables are already fixed by the operating regime of the load, the circulating current $i_{s}$ provides the freedom to optimize the MMC operation. In correspondence with the currents and voltages (10)-(14), the general form of a periodic circulating current $\underline{i}_{s}$ is given by the complex series

$$
\underline{i}_{s}=\sum_{h \in K} \underline{I}_{s}^{[h]} \mathrm{e}^{j h \theta}, \quad \text { with } \quad K \subseteq \mathbb{Z} .
$$

However, not all of the harmonics in (15) result in symmetric arm currents, which is investigated in the next section.

\subsection{Conditions and Assumptions for Symmetric MMC Operation}

During symmetric MMC operation the waveforms of the arm variables, as defined in Figure $1 b$, are phase-shifted by $\frac{\pi}{3}$ and cycle through the sequence $1,6,3,2,5,4$. Starting 
with the arm current $i_{z 1}$ of the first arm, for example, the other arm currents must satisfy the condition

$$
i_{z k}=i_{z 1}\left(\theta-\frac{\pi}{3}\left(k+(-1)^{k}\right)\right),
$$

in which $k=2, \ldots, 6$ indicates the other arms. As expected, the arm currents given in Table 2 consist of three terms each depending on the DC current $i_{s 0}$, the AC current $\underline{i}$, and the circulating current $i_{s}$, respectively. Since the arm current terms associated with the DC current $i_{s 0}$ and the AC current $\underline{i}$ from the stationary symmetric regime (10)-(14) already satisfy the symmetry condition (16), it is sufficient to focus on the arm current components as implied by the complex circulating current $\underline{i}_{s}$. Consider a particular harmonic $h$ of the circulating current definition (15). From Table 2, the contribution of this harmonic to each arm current is given by

$$
i_{z k}^{S}=\frac{1}{2} \operatorname{Re}\left(\mathrm{e}^{-j(p-1) \frac{2}{3} \pi} \underline{I}_{s}^{[h]} \mathrm{e}^{j h \theta}\right), \quad p=\left\lceil\frac{k}{2}\right\rceil, \quad k=1, \ldots, 6 .
$$

For symmetrical operation, the arm current contributions of all other arms, after reverting the phase-shift requirement (16), need to be identical to the contribution of the first arm:

$$
\begin{aligned}
i_{z 1}^{s}(\theta) & =\frac{1}{2} \operatorname{Re}\left(\underline{I}_{s}^{[h]} \mathrm{e}^{j h \theta}\right) \\
& =i_{z k}^{s}\left(\theta+\frac{\pi}{3}\left(k+(-1)^{k}\right)\right) \\
& =\frac{1}{2} \operatorname{Re}\left(\mathrm{e}^{-j(p-1) \frac{2}{3} \pi} \underline{I}_{s}^{[h]} \mathrm{e}^{j h\left(\theta+\frac{\pi}{3}\left(k+(-1)^{k}\right)\right)}\right), \quad p=\left\lceil\frac{k}{2}\right\rceil, \quad k=2, \ldots, 6 .
\end{aligned}
$$

As this restriction must hold for all $\theta \in \mathbb{R}$, (18) simplifies to

$$
2 \pi l=-(p-1) \frac{2}{3} \pi+h \frac{\pi}{3}\left(k+(-1)^{k}\right), \quad p=\left\lceil\frac{k}{2}\right\rceil, \quad k=2, \ldots, 6,
$$

in which the integer $l \in \mathbb{Z}$ provides for the periodic nature of the complex exponential. The phase index $p$ can be replaced by

$$
p=\left\lceil\frac{k}{2}\right\rceil=\frac{k}{2}+\frac{1-(-1)^{k}}{4}
$$

which allows for further simplifications in (19), leading to

$$
12 l-3=(2 h+1)(-1)^{k}+2 k(h-1), \quad k=2, \ldots, 6 .
$$

Since the harmonic order $h$ and the variable $l$ must be integers, the conditions (21) can be rewritten in the form of linear Diophantine equations

$$
a l+b h=c, \quad a=12, \quad b=-2\left((-1)^{k}+k\right), \quad c=(-1)^{k}-2 k+3, \quad k=2, \ldots, 6
$$

in $h$ and $l$. For a given $k$, a particular solution $h=h_{p}, l=l_{p}$ of (22) can be obtained by the extended Euclidean algorithm. The solutions of (22) for $h$ take the form

$$
h=h_{p}+z \frac{b}{\operatorname{gcd}(a, b)},
$$

in which $z \in \mathbb{Z}$ is any integer. The most restrictive requirements result from the cases $k=4$ or $k=6$ of the Diophantine equations (22), whose solutions are

$$
h=6 z+4=\{\ldots,-8,-2,4,10,16, \ldots\}
$$


and whose solutions are contained in the solutions to the other cases. In conclusion, the harmonics (24) of the circulating current (15) satisfy the conditions for symmetric operation and are thus available for optimization. A positive harmonic $h>0$ indicates a positive sequence circulating current, while a negative harmonic $h<0$ indicates a negative sequence. Sometimes, the considered harmonics are given as positive numbers, i.e., without the sign to indicate the respective sequence [18]. In these cases, additional measures, e.g., in the current definition, for each arm are necessary to ensure the required sequence.

Here, the circulating current in stationary operation takes the form

$$
\underline{i}_{s}=\underline{I}_{s}^{[-2]} \mathrm{e}^{-j 2 \theta}+\underline{I}_{s}^{[4]} \mathrm{e}^{j 4 \theta},
$$

i.e., the 2 nd and the 4 th harmonic are utilized for the Pareto frontier calculation in Section 3 because higher order harmonics may lie beyond the bandwidth of the current controller. Moreover, the damping nature of the cell capacitance already reduces any higher frequency arm power that might exist, rendering its compensation impractical. Inserting the circulating current definition (25) and stationary regime (10)-(14) into the inverse transform in Table 2, the current of the first arm is

$$
i_{z 1}=\frac{I_{s 0}}{2}+\frac{\hat{I}}{2} \cos (\theta+\phi)+\frac{\hat{I}_{s}^{[-2]}}{2} \cos \left(-2 \theta+\phi^{[-2]}\right)+\frac{\hat{I}_{s}^{[4]}}{2} \cos \left(4 \theta+\phi^{[4]}\right)
$$

with the abbreviations

$$
\begin{aligned}
\hat{I} & =|\underline{I}| & \phi & =\arg (\underline{I}) \\
\hat{I}_{s}^{[h]} & =\left|\underline{I}_{s}^{[h]}\right| & \phi^{[h]} & =\arg \left(\underline{I}_{s}^{[h]}\right), \quad h=-2,4 .
\end{aligned}
$$

After inserting (25) and (10)-(14), the corresponding stationary operating regimes of the energy system (6)-(9) can be found via integration, yielding

$$
\begin{aligned}
e_{s 0} & =E_{s 0} \\
e_{d 0} & =\operatorname{Re}\left(\underline{E}_{d 0}^{[3]} \mathrm{e}^{j 3 \theta}\right) \\
\underline{e}_{s} & =\underline{E}_{s}^{[-2]} \mathrm{e}^{-j 2 \theta}+\underline{E}_{s}^{[4]} \mathrm{e}^{j 4 \theta} \\
\underline{e}_{d} & =\underline{E}_{d}^{[-5]} \mathrm{e}^{-j 5 \theta}+\underline{E}_{d}^{[1]} \mathrm{e}^{j \theta}+\underline{E}_{d}^{[7]} \mathrm{e}^{j 7 \theta}
\end{aligned}
$$

with the complex constants $\underline{E}_{d 0}, \underline{E}_{s}$, and $\underline{E}_{d}$ as shown in Table 3 . The real constant $E_{s 0}$ determines the stored energy and must be chosen large enough in order to prevent saturation of the duty cycles $q_{k}$ on the upper limit of the allowed range $q_{k} \in[0,1], k=1, \ldots, 6$. As the following analysis assumes symmetrical regimes, only the first arm is considered since the arms differ only by a phase shift of their variables.

Table 3. Constants of the energies (29)-(32) for the circulating current (25) and the operating conditions (10)-(14).

\begin{tabular}{lc}
\hline$j \omega \underline{E}_{d 0}^{[3]}$ & $-\frac{2}{3} \underline{V}_{y 0}^{[3]} I_{s 0}-\frac{1}{3} \hat{V}_{y} \underline{I}_{s}^{[-2]^{*}}-\frac{1}{3} \hat{V}_{y} \underline{I}_{s}^{[4]}$ \\
\hline$j \omega \underline{E}_{s}^{[-2]}$ & $-\frac{1}{2} V_{\mathrm{DC}} \underline{I}_{s}^{[-2]}+\frac{1}{2} \hat{V}_{y} \underline{I}^{*}+\frac{1}{2} \underline{\underline{I}} \underline{V}_{y 0}^{[3]^{*}}$ \\
$j \omega \underline{E}_{s}^{[4]}$ & $\frac{1}{4} V_{\mathrm{DC}} \underline{I}_{s}^{[4]}-\frac{1}{4} \underline{I} \underline{V}_{y 0}^{[3]}$ \\
\hline$j \omega \underline{E}_{d}^{[-5]}$ & $\frac{1}{5} \underline{I}_{s}^{[-2]} \underline{V}_{y 0}^{[3]^{*}}+\frac{1}{5} \underline{I}_{s}^{[4]^{*}} \hat{V}_{y}$ \\
$j \omega \underline{E}_{d}^{[1]}$ & $V_{\mathrm{DC}} \underline{I}-2 I_{s 0} \hat{V}_{y}-\underline{I}_{s}^{[-2]^{*}} \hat{V}_{y}-\underline{I}_{s}^{[-2]} \underline{V}_{y 0}^{[3]}-\underline{I}_{s}^{[4]} \underline{V}_{y 0}^{[3]^{*}}$ \\
$j \omega \underline{E}_{d}^{[]]}$ & $-\frac{1}{7} \underline{I}_{s}^{[4]} \underline{V}_{y 0}^{[3]}$ \\
\hline
\end{tabular}




\subsection{Cell Capacitor Design}

The MMC cell capacitor design can be based on the equivalent cell when the voltage inequality between individual cells is neglected or taken into account by a voltage margin. Considering the minimum and maximum voltages $v_{\mathrm{C} 1 \text {, min }}$ and $v_{\mathrm{C} 1 \text {, max }}$ and the corresponding minimum and maximum capacitor energies $e_{\mathrm{C} 1, \min }$ and $e_{\mathrm{C} 1 \text {, max }}$, results in the equivalent cell capacitance design equation

$$
C=2 \frac{\Delta e_{C 1}}{v_{C 1, \max }^{2}-v_{C 1, \min }^{2}}, \quad \Delta e_{C 1}=e_{C 1, \max }-e_{C 1, \min }
$$

The energy ripple $\Delta e_{C 1}$ is determined by the stationary operating regime under consideration, while the minimum and maximum voltages in (33) must be chosen appropriately. Usually, the maximum voltage $v_{\mathrm{C} 1 \text {, max }}$ is selected first because it is closely linked to the power semiconductor voltage utilization in stationary operation and to the number of cells per $\operatorname{arm} n$. For the minimum cell voltage $v_{\mathrm{C} 1, \mathrm{~min}}$, three cases can be distinguished as depicted in Figure 2:

(a) $v_{\mathrm{C} 1 \text {, min }}$ is set to the $\mathrm{DC}$ voltage $v_{\mathrm{DC}}$, so each arm is capable of blocking the DC voltage.

(b) $v_{\mathrm{C} 1, \mathrm{~min}}$ is set to the maximum injected arm voltage $v_{q 1 \text { max }}$ irrespective of its location.

(c) $v_{C 1, \min }$ is set to the lowest possible value until all the available arm voltage is used.

The first approach implies a considerable voltage reserve at the upper limits [1] The second approach increases the arm voltage utilization without endangering the control reserve. The last approach is applied in [31,32] considering regimes with zero commonmode voltage. The solution in [22] uses the common-mode voltage to evade the impending arm voltage saturation at the upper limit allowing for a further capacitance reduction in the case of the third approach. However, this evasion reduces the arm voltage margin on the lower limit given by the half-bridge cells because it manipulates the 3rd and 9th harmonic of the common-mode voltage and moves the minimum of the required arm voltages $v_{q 1}$ closer to zero. In this paper, the injected arm voltage is fixed in each operating regime because the common-mode voltage is set by (12) in order to maintain a good DC voltage utilization. Therefore, any of the minimum cell voltage selections is feasible, and the required capacitance can still be reduced significantly because the energy ripple $\Delta e_{C 1}$ can be lowered using circulating currents.

The calculations can be simplified when the equivalent cell energy ripple $\Delta e_{C 1}$ is approximated by

$$
\Delta e_{C 1}=\Delta e_{z 1}
$$

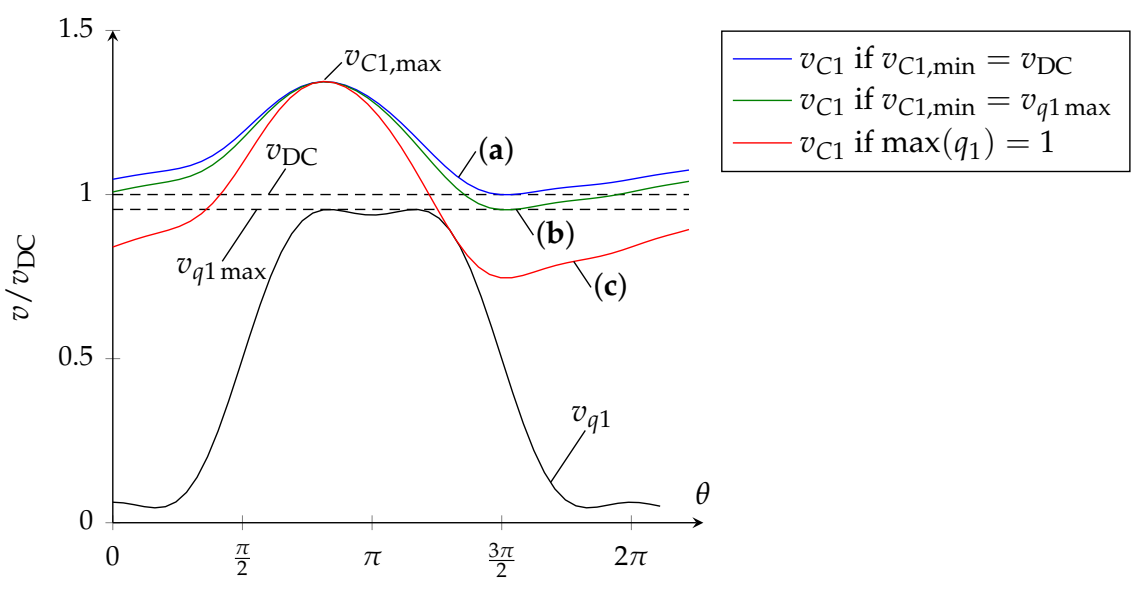

Figure 2. Three capacitor designs for the same energy ripple $\Delta e_{C 1}$ and maximum cell voltage $v_{C 1, \max }$ but different choices for the minimum voltage $v_{C 1 \text {, min }}$, leading to different voltage ripples $\Delta v_{C 1}$ : (a) the minimum voltage equals the DC voltage; (b) the minimum voltage equals the maximum injected arm voltage; (c) the minimum is the lowest possible value satisfying $v_{q 1} \leq v_{C 1}$. 
in which $\Delta e_{z 1}$ is the arm energy ripple

$$
\Delta e_{z 1}=e_{z 1, \max }-e_{z 1, \min } .
$$

In a typical MMC design, the stored energy in the arm inductance is much lower compared to the stored energy in the equivalent cell capacitance making (34) a good approximation. Given that the arm inductance is not extremely large, the relation (34) is even exact because the arm energy extrema coincide with the extrema of the equivalent cell voltage because they happen to be at the zero crossings of the arm current.

\subsection{Arm Conduction Losses}

For periodic stationary operating regimes, the power device conduction losses can be determined by

$$
P_{\text {cond }}=R_{0} I_{\text {rms }}^{2}+V_{0} I_{\text {avg }},
$$

in which $R_{0}$ and $V_{0}$ are parameters of a piecewise linear approximation of the on-state characteristics, while $I_{\text {rms }}$ and $I_{\text {avg }}$ are the rms and mean values of the device current in a forward direction. In general, the parameters $R_{0}$ and $V_{0}$ are different for the diodes and the Insulated Gate Bipolar Transistors (IGBTs), complicating the calculations especially in cases when only the total conduction losses are of interest. Therefore, and, in the following, the mean of the diode and IGBT parameters will be used, leading to

$$
R_{0}=\frac{R_{0 \text { (diode) }}+R_{0(\mathrm{IGBT})}}{2} \text { and } V_{0}=\frac{V_{0 \text { (diode) }}+V_{0(\mathrm{IGBT})}}{2} .
$$

By the above simplifications, the total arm conduction losses can be expressed as

$$
P_{z 1}=R_{z} i_{z 1, \mathrm{rms}}^{2}+V_{T z}\left|i_{z 1}\right|_{\mathrm{avg}}
$$

in which $R_{z}$ is the lumped arm series resistance, including $n R_{0}$ to represent all $n$ cells, while $V_{T z}=n V_{0}$ represents the lumped part of the forward voltage drop that is current independent. Unsurprisingly, the conduction losses (38) depend on the rms of the arm current, denoted by $i_{z 1, \text { rms }}$. In addition, since the arm current replaces the device forward current in the conduction losses (38), the arm current average rectified value

$$
\left|i_{z 1}\right|_{\text {avg }}=\frac{1}{2 \pi} \int_{0}^{2 \pi}\left|i_{z 1}(\theta)\right| \mathrm{d} \theta
$$

is to be calculated. On the one hand, the averaging of power device forward characteristics in (37) reduces accuracy, but, on the other hand, enables a lumped losses approximation. In contrast, the losses calculation in [33] uses a weighted average of diode and IGBT parameters, but depends on the duty cycles.

Although the assumed equivalent circuit in Figure $1 \mathrm{~b}$ and the energy dynamics (6)-(9) neglect losses, the predicted arm currents can be used for the losses expression (38).

\section{Pareto Frontier Calculation}

For a multiple-objective optimization problem, it is generally not possible to simultaneously optimize each objective as their solutions may be competing. In particular, during MMC operation in stationary regimes, the minimization of both the energy ripple and the arm power losses are such competing objectives.

One possible solution is to introduce a weighting factor between both and to solve the resulting minimization problem. However, this raises the problem of finding a suitable weighting factor. In order to avoid this, it seems more helpful to identify the set of parametrizations for the circulating current that dominate others such that each point in the set cannot be improved in one objective without worsening the other. These points are called Pareto-optimal solutions and the set itself is termed the Pareto frontier. From the Pareto frontier, the decision-maker can choose the best solution depending on the desired 
trade-off or on the underlying constraints. In an MMC application, the optimal compromise between the energy ripple and arm losses will depend on the cooling requirements, capacitor size, semiconductor rating, among others.

In the next section, a method to calculate the Pareto frontier is introduced. In order to improve the computation speed, expressions to efficiently calculate the energy ripple and arm losses, as well as their gradients, are derived.

\subsection{Objective Function}

The set of Pareto optimal solutions can be calculated solving the minimization problem:

$$
\min _{\hat{I}_{s}^{[-2]}, \phi^{[-2],} \hat{I}_{s}^{[4]}, \phi^{[4]}} F=\min _{\hat{I}_{s}^{[-2]}, \phi^{[-2]}, \hat{I}_{s}^{[4]}, \phi^{[4]}}\left\{\lambda \frac{\Delta e_{z 1}}{E_{s}}+(1-\lambda) \frac{P_{z 1}}{P_{s}}\right\}
$$

for $\lambda \in[0,1]$, in which $\lambda$ is a weighting factor that selects a particular optimal solution of the multi-objective optimization problem [34]. The constants $E_{s}$ and $P_{s}$ are used to obtain a dimensionless quantity.

In order to numerically minimize (40), $\Delta e_{z 1}$ and $P_{z 1}$ must be efficiently calculated in each iteration. As for $\Delta e_{z 1}$, the arguments of its local extrema can be found by differentiating (1) with respect to $\theta$ and setting this derivative to zero

$$
\frac{\mathrm{d}}{\mathrm{d} \theta} e_{z 1}=\left(v_{q 1}+L_{z} \frac{\mathrm{d}}{\mathrm{d} \theta} i_{z 1}\right) i_{z 1}=0 .
$$

From (41), it can be found that the energy is maximum or minimum at the zerocrossing angles of $i_{z 1}$, as the arm voltage is positive for all $\theta \in \mathbb{R}$. For the calculation of the roots of $i_{z 1}$, the expression in (26) must be transformed into a polynomial of degree eight.

Let $2 v=\theta, a=\cos (v)$, and $x=\tan (v)$. Combining the Euler formula and the binomial theorem [35], the following general expression for $\cos (n v+\varphi)$ is derived:

$$
\begin{aligned}
\cos (n v+\varphi) & =a^{n} \frac{(1+j x)^{n} e^{j \varphi}+(1-j x)^{n} e^{-j \varphi}}{2} \\
& =a^{n} \sum_{k=0}^{n}\left(\begin{array}{c}
n \\
n-k
\end{array}\right) x^{k} \cos \left(\varphi+\frac{k}{2} \pi\right) \\
& =a^{n} P_{n}(x, \varphi)
\end{aligned}
$$

where $P_{n}(x, \varphi)$ is a polynomial of degree $n$ in $x$ for all $\varphi \in \mathbb{R}$. Substituting (44) in each cosine term of (26), setting the current to zero, and utilizing the identity $a^{2}\left(1+x^{2}\right)=1$ yields

$$
\begin{aligned}
0 & =a^{8}\left(I_{s 0}\left(1+x^{2}\right)^{4}+\hat{I}\left(1+x^{2}\right)^{3} P_{2}(x, \phi)+\hat{I}_{s}^{[-2]}\left(1+x^{2}\right)^{2} P_{4}\left(x,-\phi^{[-2]}\right)+\hat{I}_{s}^{[4]} P_{8}\left(x, \phi^{[4]}\right)\right) \\
& =a^{8} P_{I_{z 1}}(x)
\end{aligned}
$$

where $P_{I_{z 1}}(x)$ is a polynomial in $x$ of degree eight, whose roots are used to calculate the zero-crossing angles

$$
\left\{z_{1}, \ldots, z_{Q}\right\}=\{2 \arctan (x)+2 \pi k \mid 0 \leq \arctan (x)+\pi k<\pi, k \in \mathbb{Z}\}
$$

in which $Q \in\{2, \ldots, 8\}$ corresponds to the number of real roots of which at least two are assumed to exist. Thus, $\Delta e_{z 1}$ is calculated in each iteration as

$$
\Delta e_{z 1}=\max _{\theta \in\left\{z_{1}, \ldots, z_{Q}\right\}}\left\{e_{z 1}(\theta)\right\}-\min _{\theta \in\left\{z_{1}, \ldots, z_{Q}\right\}}\left\{e_{z 1}(\theta)\right\}
$$


and $e_{z 1}(\theta)$ is given by

$$
e_{z 1}(\theta)=\frac{E_{s 0}}{4}+\frac{1}{4} \operatorname{Re}\left(\sum_{n \in H} \underline{E}_{z 1}^{[n]} e^{j n \theta}\right)
$$

where $H=\{1,-2,3,4,-5,7\}$, and $\underline{E}_{z 1}^{[n]}$ denotes the complex coefficients of the exponential Fourier series (29)-(32) given in Table 3. Assuming $m=0, I_{s 0}=0, \hat{I}_{s}^{[-2]}=0$, and $\hat{I}_{s}^{[4]}=0$, the energy ripple (48) becomes

$$
\Delta e_{z 1}=\frac{V_{\mathrm{DC}} \hat{I}}{2 \omega}=: E_{s}
$$

which provides a suitable setting for the energy scaling constant $E_{s}$ in the objective function (40).

For the calculation of the losses, the rectified current (39) is integrated over intervals between successive zero-crossing angles

$$
\left|i_{z 1}\right|_{\text {avg }}=\sum_{i=0}^{Q} \int_{z_{i}}^{z_{i+1}} \operatorname{sign}\left(i_{z 1}(\theta)\right) i_{z 1}(\theta) \mathrm{d} \theta
$$

in which $z_{0}=0$ and $z_{Q+1}=2 \pi$. Since the sign of the current is constant on each interval, its argument is replaced with the midpoint and factored out of the integrand, and then the current is analytically integrated. Inserting (51) into (38), the losses are calculated in each iteration as

$$
P_{z 1}=\frac{R_{z}}{8}\left(2 I_{s 0}^{2}+\hat{I}^{2}+\left(\hat{I}_{s}^{[-2]}\right)^{2}+\left(\hat{I}_{s}^{[4]}\right)^{2}\right)+\frac{V_{T z}}{2 \pi} \sum_{i=0}^{Q} \operatorname{sign}\left(i_{z 1}\left(\frac{z_{i}+z_{i+1}}{2}\right)\right) \int_{z_{i}}^{z_{i+1}} i_{z 1}(\theta) \mathrm{d} \theta .
$$

Assuming $m=0, I_{s 0}=0, \hat{I}_{s}^{[-2]}=0$, and $\hat{I}_{s}^{[4]}=0$, the conduction losses (52) simplify to

$$
P_{z 1}=\frac{R_{z} \hat{I}^{2}}{8}+\frac{V_{T z} \hat{I}}{\pi}=: P_{s},
$$

which provides a suitable setting for the losses scaling constant $P_{S}$ in the objective function (40).

\subsection{Gradient Calculation}

In order to improve the convergence and computation speed, the gradient of the objective function $F$ is derived. Due to its linear property, the gradient of $F$ is calculated as the sum of the energy ripple and losses gradients.

For the energy ripple gradient calculation, (48) is differentiated with respect to $X \in$ $\left\{\hat{I}^{[-2]}, \phi^{[-2]}, \hat{I}^{[4]}, \phi^{[4]}\right\}$, obtaining

$$
\frac{\mathrm{d}}{\mathrm{d} X} \Delta e_{z 1}=\frac{\mathrm{d}}{\mathrm{d} X} e_{z 1}\left(z_{e-\max }\right)-\frac{\mathrm{d}}{\mathrm{d} X} e_{z 1}\left(z_{e-\min }\right),
$$

where $z_{e-\max }$ and $z_{e-\min }$ correspond to the roots in which the energy is maximum and minimum, respectively. Considering that the roots depend on $X$, the energy (49) is evaluated at an arbitrary root $z$, and then differentiated applying the chain rule, yielding

$$
\frac{\mathrm{d}}{\mathrm{d} X} e_{z 1}(z)=\frac{1}{4} \operatorname{Re}\left(\sum_{n \in H} e^{j n z} \frac{\mathrm{d}}{\mathrm{d} X} \underline{E}_{z 1}^{[n]}\right)+\underbrace{\frac{1}{4} \operatorname{Re}\left(\sum_{n \in H} j n \underline{E}_{z 1}^{[n]} e^{j n z}\right)}_{\left.\frac{\mathrm{d}}{\mathrm{d} \theta} e_{z 1}\right|_{z=0}} \frac{\mathrm{d}}{\mathrm{d} X} z
$$


where the second term of the right-hand side of (55) is canceled, as one of its factors corresponds to the derivative of the energy evaluated in $z$, which is zero by definition.

Regarding the losses gradient calculation, (52) is differentiated with respect to $X$ and the Leibniz rule is applied to the integral term

$$
\frac{\mathrm{d}}{\mathrm{dX}} \int_{z_{i}}^{z_{i+1}} i_{z 1}(\theta) \mathrm{d} \theta=i_{z 1}\left(z_{i+1}\right) \frac{\mathrm{d}}{\mathrm{d} X} z_{i+1}-i_{z 1}\left(z_{i}\right) \frac{\mathrm{d}}{\mathrm{d} X} z_{i}+\int_{z_{i}}^{z_{i+1}} \frac{\mathrm{d}}{\mathrm{d} X} i_{z 1}(\theta) \mathrm{d} \theta,
$$

noting that the first two terms of the right-hand side of (56) are zero, as $i_{z 1}\left(z_{i}\right)=0$ for $i \in[1, Q]$ and $\frac{\mathrm{d}}{\mathrm{dX}} z_{0}=\frac{\mathrm{d}}{\mathrm{dX}} z_{Q+1}=0$, which leads to

$$
\begin{aligned}
\frac{\mathrm{d}}{\mathrm{d} X} P_{z 1}= & \frac{R_{z}}{4}\left(\hat{I}_{s}^{[-2]} \frac{\mathrm{d}}{\mathrm{d} X} \hat{I}_{s}^{[-2]}+\hat{I}_{s}^{[4]} \frac{\mathrm{d}}{\mathrm{d} X} \hat{I}_{s}^{[4]}\right) \\
& +\frac{V_{T z}}{2 \pi} \sum_{i=0}^{Q} \operatorname{sign}\left(i_{z 1}\left(\frac{z_{i}+z_{i+1}}{2}\right)\right) \int_{z_{i}}^{z_{i+1}} \frac{\mathrm{d}}{\mathrm{d} X} i_{z 1}(\theta) \mathrm{d} \theta .
\end{aligned}
$$

Finally, each component of the gradient of F is calculated by combining (55) and (57) as follows:

$$
\frac{\mathrm{d}}{\mathrm{d} X} F=\frac{\lambda}{E_{S}} \frac{\mathrm{d}}{\mathrm{d} X} \Delta e_{z 1}+\frac{1-\lambda}{P_{S}} \frac{\mathrm{d}}{\mathrm{dX}} P_{z 1}
$$

\subsection{Pareto Frontier Symmetries}

From the energy ripple and arm losses definitions, it is possible to notice an invariance with respect to the sign of the current and inversion of time, leading to symmetries in the Pareto frontier. Furthermore, these properties can be utilized to reduce the load angle search space, avoiding additional calculations.

On the one hand, it is easy to verify that $\left|i_{z}\right|_{\text {avg }}=\left|-i_{z}\right|_{\text {avg }}$ and $\left(-i_{z}\right)_{\mathrm{rms}}=i_{z, \mathrm{rms}}$. On the other hand, flipping the arm current waveform with respect to the $y$-axis gives a flipped arm power waveform, which has the same energy ripple. Thus, it can be concluded that the sign of the current neither affects the losses nor the energy ripple. In a similar approach, it is easy to verify that an inversion of time $\theta^{\prime}=-\theta$ leads to flipped versions of the arm current and power waveforms with respect to the $x$-axis, resulting in the same losses and energy ripple values. Taking into account these properties, symmetries in solutions of the Pareto frontier are derived in the following.

Let $X=\left\{\phi_{2}, \phi_{4}, \hat{I}_{s 2}, \hat{I}_{s 4}\right\}$ be a known solution of the Pareto frontier for parameters $\lambda$, $V_{D C}, \hat{V}_{y}, \hat{I}$, and $\phi$; and $X^{\prime}=\left\{\phi_{2}^{\prime}, \phi_{4}^{\prime}, \hat{I}_{s 2}^{\prime}, \hat{I}_{s 4}^{\prime}\right\}$ an unknown solution for $\lambda, V_{D C}, \hat{V}_{y}, \hat{I}$, and $\phi^{\prime}=-\phi$. The goal is to utilize the solution $X$ for $\phi$ to calculate $X^{\prime}$ for $\phi^{\prime}=-\phi$. Consider the current $i_{z 1}^{\prime}\left(\theta^{\prime}\right)$

$i_{z 1}^{\prime}\left(\theta^{\prime}\right)=\frac{\hat{I} \hat{V}_{y}}{2 V_{D C}} \cos \left(\phi^{\prime}\right)+\frac{\hat{I}}{2} \cos \left(\theta^{\prime}+\phi^{\prime}\right)+\frac{\hat{I}_{s}^{[-2]^{\prime}}}{2} \cos \left(2 \theta^{\prime}-\phi^{[-2]^{\prime}}\right)+\frac{\hat{I}_{s}^{[4]^{\prime}}}{2} \cos \left(4 \theta^{\prime}+\phi^{[4]^{\prime}}\right)$.

Substituting $\phi^{\prime}=-\phi$ and applying a time inversion to (59), the current can be expressed as

$$
i_{z 1}^{\prime}(\theta)=\frac{\hat{I} \hat{V}_{y}}{2 V_{D C}} \cos (\phi)+\frac{\hat{I}}{2} \cos (\theta+\phi)+\frac{\hat{I}_{s}^{[-2]^{\prime}}}{2} \cos \left(2 \theta+\phi^{[-2]^{\prime}}\right)+\frac{\hat{I}_{s}^{[4]^{\prime}}}{2} \cos \left(4 \theta-\phi^{[4]^{\prime}}\right) .
$$

By comparison with the form (26) of the original solution, it is possible to deduce that $\hat{I}_{s}^{[-2]^{\prime}}=\hat{I}_{s}^{[-2]}, \hat{I}_{s}^{[4]^{\prime}}=\hat{I}_{s}^{[4]}, \phi^{[-2]^{\prime}}=-\phi^{[-2]}$, and $\phi^{[4]^{\prime}}=-\phi^{[4]}$, which corresponds to a reflection with respect to $\phi=0$. 
A second symmetry can be derived assuming $\phi^{\prime}=\pi-\phi$, where, after applying a time inversion and a change of sign to (59), the current leads to

$$
\begin{aligned}
i_{z 1}(\theta)= & \frac{\hat{I} \hat{V}_{y}}{2 V_{D C}} \cos (\phi)+\frac{\hat{I}}{2} \cos (\theta+\phi)+\frac{\hat{I}_{s}^{[-2]^{\prime}}}{2} \cos \left(2 \theta+\phi^{[-2]^{\prime}}+\pi\right) \\
& +\frac{\hat{I}_{s}^{[4]^{\prime}}}{2} \cos \left(4 \theta-\phi^{[4]^{\prime}}+\pi\right) .
\end{aligned}
$$

Again, by comparison with the form (26) of the original solution, it is deduced that $\hat{I}_{s}^{[-2]^{\prime}}=\hat{I}_{s}^{[-2]}, \hat{I}_{s}^{[4]^{\prime}}=\hat{I}_{s}^{[4]}, \phi^{[-2]^{\prime}}=\pi-\phi^{[-2]}$, and $\phi^{[4]^{\prime}}=\pi-\phi^{[4]}$, corresponding to a reflection with respect to $\phi=\frac{\pi}{2}$.

Finally, combining both reflections, it is deduced that, for $\phi^{\prime}=\pi+\phi$, the solution is given by $\hat{I}_{s}^{[-2]^{\prime}}=\hat{I}_{s}^{[-2]}, \hat{I}_{s}^{[4]^{\prime}}=\hat{I}_{s}^{[4]}, \phi^{[-2]^{\prime}}=\pi+\phi^{[-2]}$, and $\phi^{[4]^{\prime}}=\pi+\phi^{[4]}$.

\subsection{Numerical Results}

To illustrate how the Pareto frontier changes with respect to the operating conditions, a low-voltage MMC, similar to the experimental setup, is numerically evaluated using the model presented in previous sections. The lumped loss parameters of the simulated model are $R_{z}=166.9 \mathrm{~m} \Omega$ and $V_{T z}=4.522 \mathrm{~V}$. The objective function and its gradient are implemented as a MATLAB script [36], and the minimization problem is solved through the Interior-point method by means of the built-in function fmincon. In a comparison, similar results were obtained with the MATLAB functions paretosearch, patternsearch, and gamultiobj. However, the Pareto frontier obtained by fmincon was superior in all the examples we tried. Nevertheless, because (40) is non-convex, it is not guaranteed that the global minimum will be found. Thus, in order to obtain a good approximation of the global minimum, and for each value of $\lambda$, the minimization algorithm is run for multiple starting points, and the solution for the lowest value of $F$ is chosen. One of the starting points corresponds to the circulating current reference given by case $C$, while the other initial points are randomly generated from the uniformly distributed interval $[0, \hat{I}]$ for $\hat{I}_{S}^{[-2]}$ and $\hat{I}_{S}^{[4]}$, and $[-\pi, \pi]$ for $\phi^{[-2]}$ and $\phi^{[4]}$. For the following results, eight starting points are evaluated for each iteration.

The algorithm is run for different modulation indexes, output current amplitudes, and displacement angles, showing in Figures 3-5 the obtained Pareto frontiers together with the cases A, B, and C from [28] for comparison. Case A sets the circulating current to zero, case B eliminates $\underline{E}_{s}^{[-2]}$ requiring only a 2nd harmonic, and case $C$ eliminates $\underline{E}_{s}^{[-2]}$ and $\underline{E}_{s}^{[4]}$. As can be seen for high values of $m$, the losses are slightly decreased with respect to zero circulating current injection, with a great reduction in energy ripple. For low values of $m$, the energy ripple range of the Pareto frontier tends to shrink, while the arm losses range stretches and then shrinks. On the other hand, as $m$ is decreased, the difference between the minimum losses operating point and the zero circulating current condition is reduced. For the extreme case $m=0$, e.g., a fault on the AC side, the Pareto frontier and cases A, B, and $C$ collapse into a single point at $(1,1)$, in which the minimum losses and energy ripple are achieved at the same time with $\underline{i}_{s}=0$. Cases B and C are pretty close to the Pareto frontier for all variations, except for an increased distance of case B when the angle $\phi$ is varied in Figure 5.

As for the amplitude of output current, results for different values of $\hat{I}$ are depicted in Figure 4. Noticeably, the minimum energy ripple is independent of $\hat{I}$, while the arm losses can be further reduced with respect to zero circulating current injection, as $\hat{I}$ is decreased.

Finally, results for different load angles are shown in Figure 5. It can be seen that, as $\phi$ increases, the Pareto frontier stretches in both axes, decreasing the arm losses and increasing the energy ripple. Due to symmetries in the Pareto frontier, as discussed in Section 3.3, results are only given for values of $\phi$ in the first quadrant, as reflections of $\phi$ in the other three quadrants lead to the same results. 


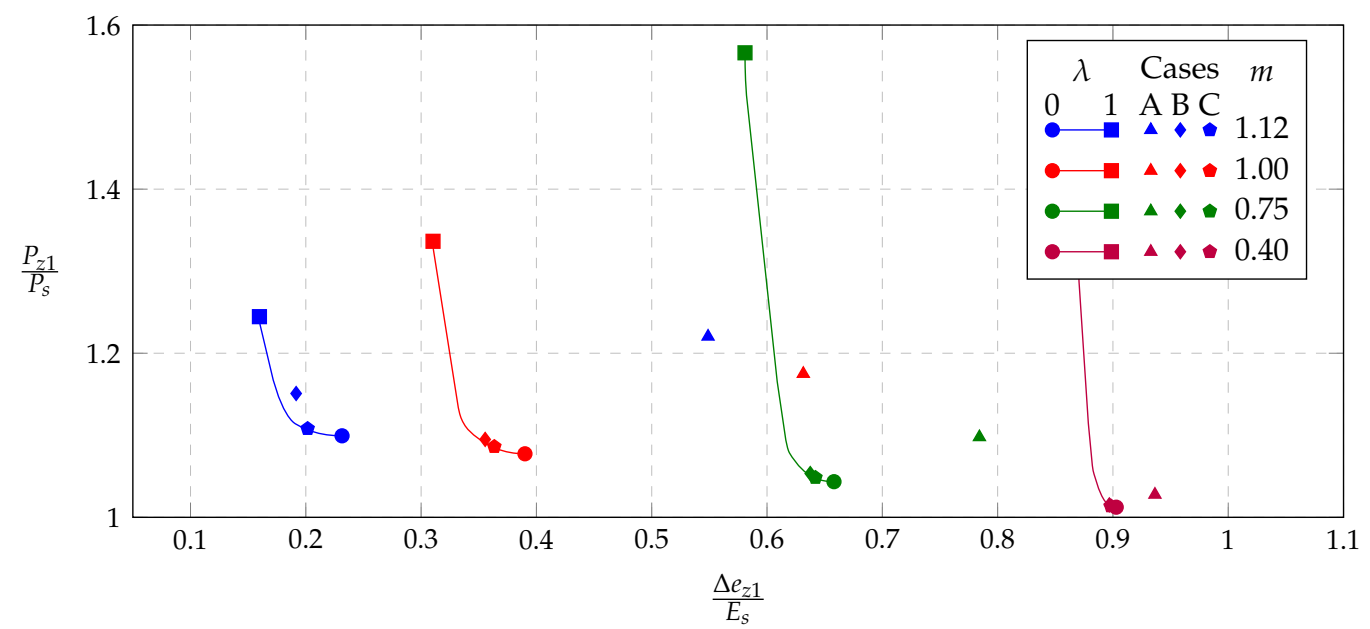

Figure 3. Pareto frontier for a low-voltage MMC with $R_{z}=166.9 \mathrm{~m} \Omega, V_{T z}=4.522 \mathrm{~V}$ and $\hat{I}=10 \mathrm{~A}$ at $\phi=0^{\circ}$ and $m \in\{0.4,0.75,1.00,1.12\}$.

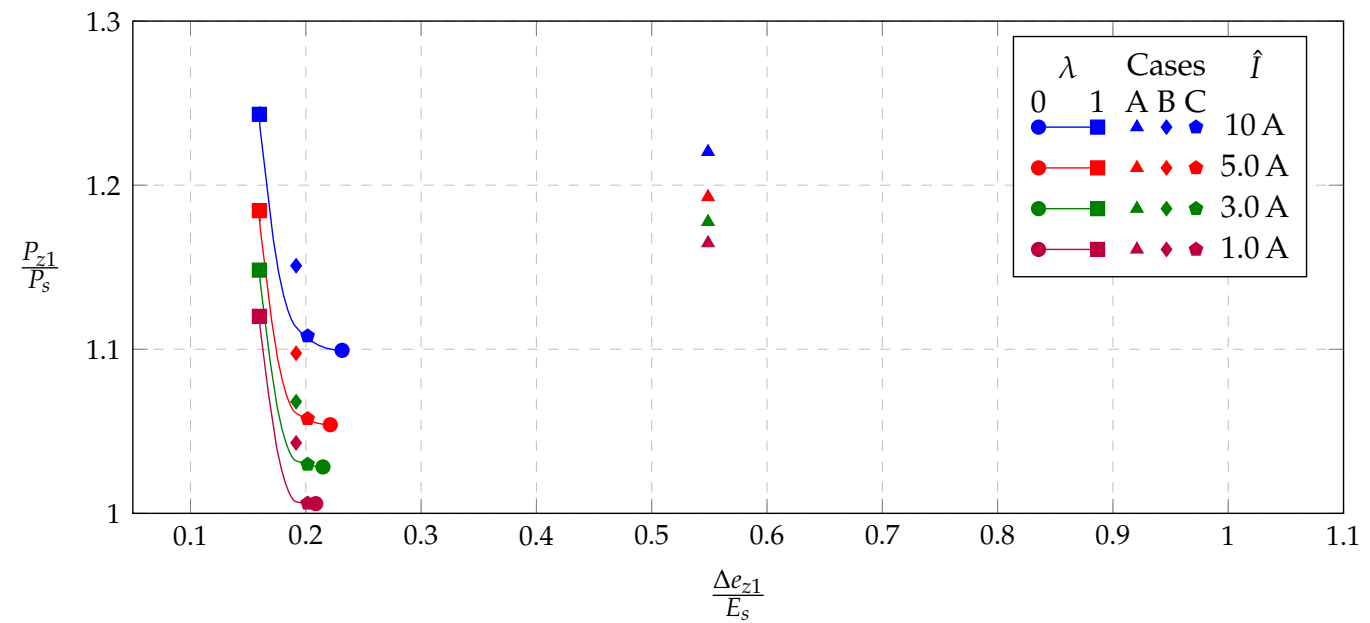

Figure 4. Pareto frontier for a low-voltage MMC with $R_{z}=166.9 \mathrm{~m} \Omega, V_{T z}=4.522 \mathrm{~V}$ and $\hat{I} \in\{10 \mathrm{~A}, 5.0 \mathrm{~A}, 3.0 \mathrm{~A}, 1.0 \mathrm{~A}\}$ at $\phi=0^{\circ}$ and $m=1.12$.

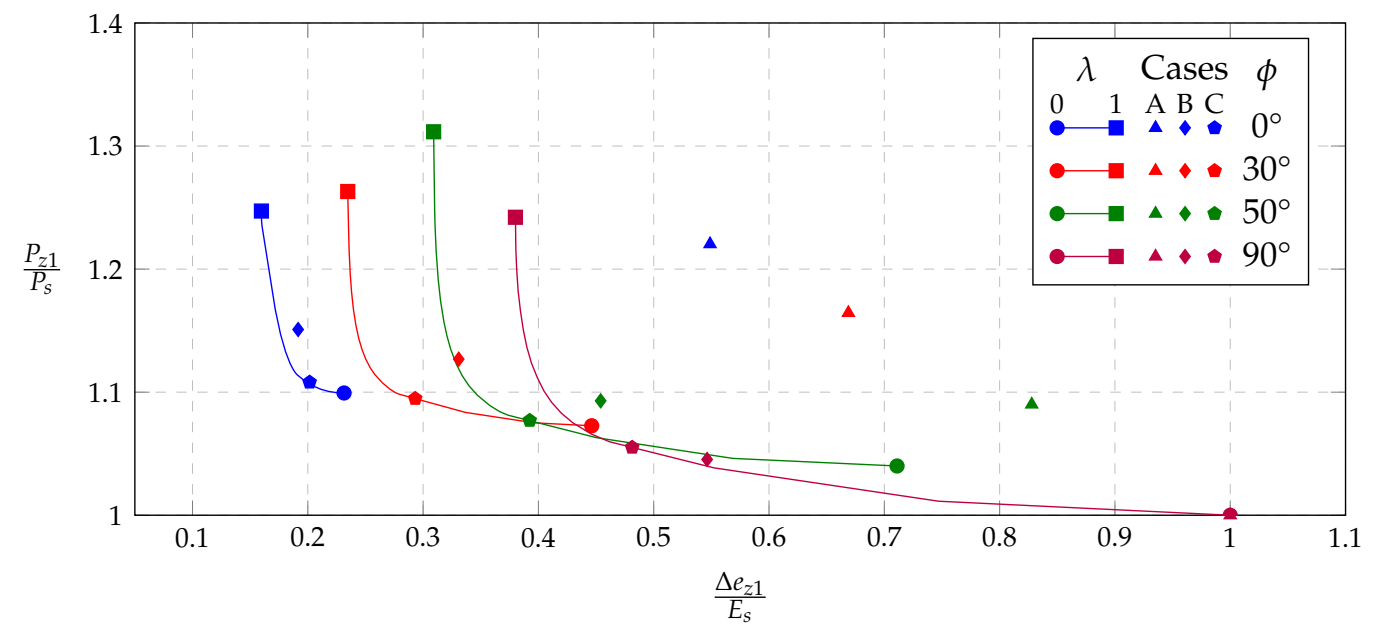

Figure 5. Pareto frontier for a low-voltage MMC with $R_{z}=166.9 \mathrm{~m} \Omega, V_{T z}=4.522 \mathrm{~V}$ and $\hat{I}=10 \mathrm{~A}$ at $\phi \in\left\{0^{\circ}, 30^{\circ}, 50^{\circ}, 90^{\circ}\right\}$ and $m=1.12$. 


\subsection{Usage of the Pareto Frontier in an MMC Design Procedure}

In conclusion, the Pareto frontier reveals the trade-off between arm energy ripple and conduction losses of an MMC. Requiring only a limited set of parameters, it can be obtained very early in the converter design procedure, without simulations. With the trade-off numerically and visually characterized, the design task is now as easy as selecting a point on the Pareto frontier that corresponds to specific values of the energy ripple and conduction losses. The impact of a particular selection on the resulting MMC design is obvious because the energy ripple is linked to the capacitor and volume requirement, while the conduction losses indicate the semiconductor cooling requirements and converter efficiency. After setting the maximum and minimum equivalent cell voltages, e.g., as described in Section 2.3, the capacitor design Equation (33) can be applied, using the relation (34), and the corresponding energy ripple from the selected point on the Pareto frontier. The feasible operating range of the resulting MMC design includes all points with lower energy ripple and lower conduction losses. Of course, some margin is to be provided for the neglected effects, such as the switching losses, unequal cell capacitor voltage due to non-ideal balancing and deviations from the symmetrical operating regime given by (10)-(14) and (25).

\section{Implementation and Experimental Results}

The Pareto optimal current references from Section 3.4 are implemented on a lowvoltage test-bench, depicted in Figure 6. The grid side MMC, whose parameters are given in Figure 1b, was connected to a grid emulator, while the 3L-VSC was used to control the DC voltage. During rectifier mode, a resistor bank (not shown) is used as the AC load for the 3L-VSC.

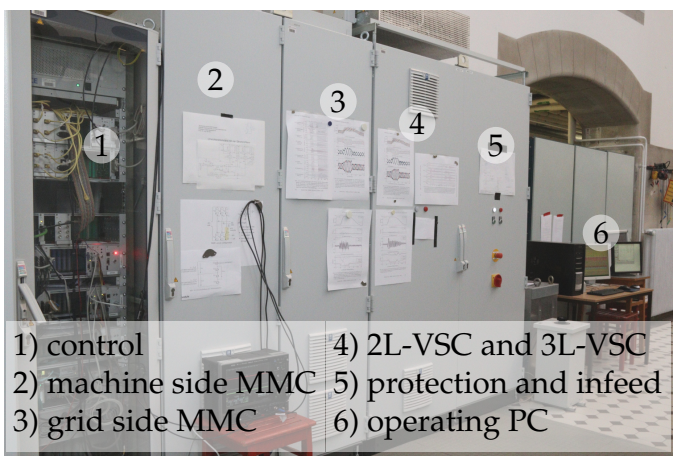

(a) overview

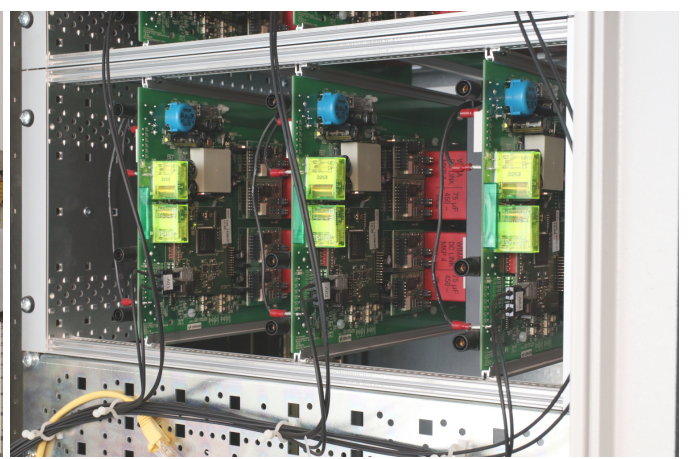

(c) grid side MMC cell boards

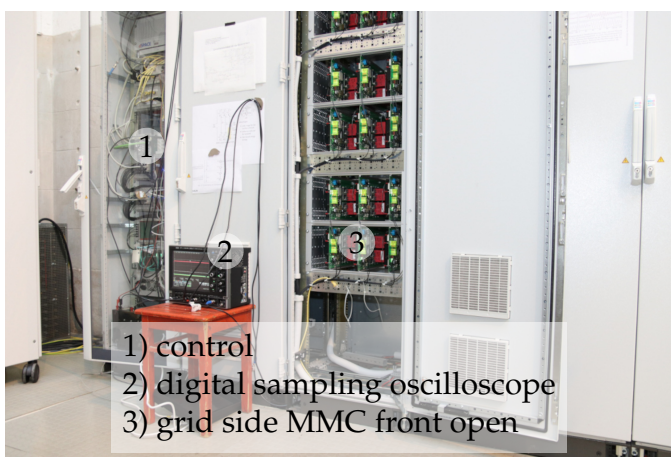

(b) grid side MMC front

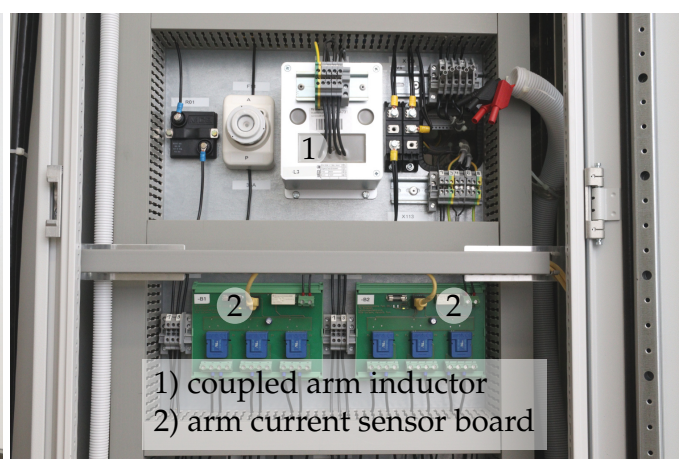

(d) grid side MMC rear

Figure 6. Pictures of the low-voltage test-bench: (a) overview, (b) MMC front view, (c) MMC cells, (d) MMC rear view. Each cell board holds two cells. Only the grid side MMC and the 3L-VSC (three-level voltage source converter) were used in the experiments. The grid emulator (Cinergia GE-AC-15) was connected via the infeed cabinet, denoted 5 in (a). 
A block diagram of the MMC control scheme including the circulating current references is given in Figure 7.

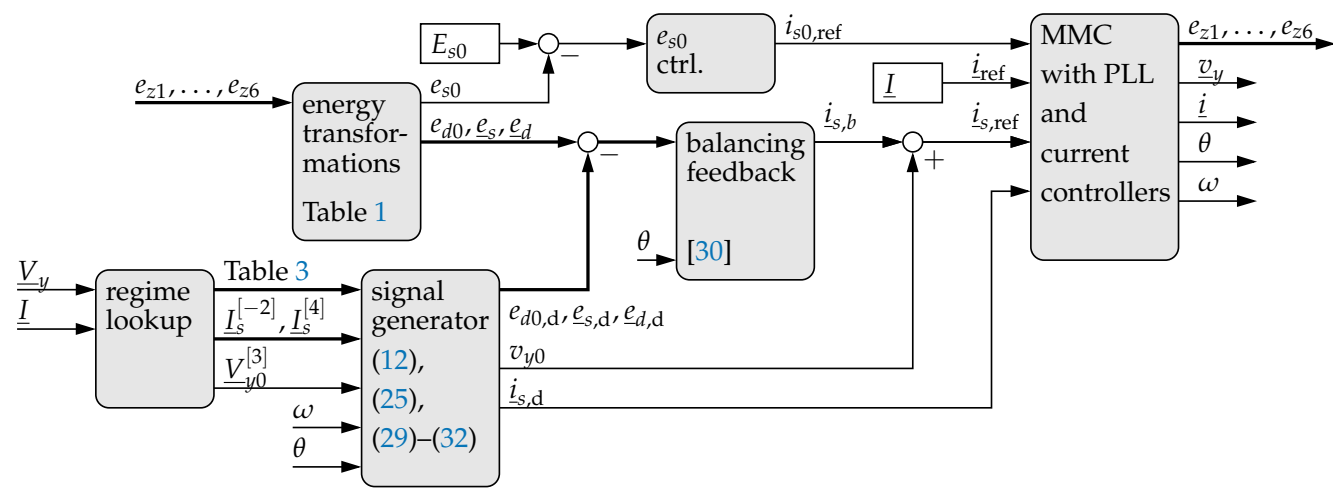

Figure 7. Block diagram of the control scheme for the experiments on a low-voltage test-bench.

Depending on the selected solution, the regime lookup block provides the respective constants that make up the stationary operation to the signal generator. By implementing the energy series (29)-(32), the circulating current definition (25) and the common-mode voltage (12), the signal generator provides the respective nominal signals which are used by the remaining blocks representing a typical MMC control scheme including a balancing feedback. In addition to the Pareto optimal circulating currents, the cases A, B, and C from [28] are implemented in the regime lookup, providing a means for comparison.

The converter control is based on a dSPACE modular real-time control prototyping system, in which the control scheme is implemented on a DS1005 processor board. Each MMC has its own custom board based on an XC6SLX150 FPGA for protection, cell voltage measurement collection, balancing, and modulation. Each cell board is linked to the custom board via a bidirectional fiber optic link, i.e., for duty cycle updates and capacitor voltage measurements. Arm currents, AC, and DC side voltages were measured utilizing interface boards for signal conditioning and isolation, and an analogue-digital DS2004 board for digital conversion. Arm current and cell voltage measurements are taken by the control system at the positive peak of the triangular carrier signal, at a sampling rate of $T=205 \mu \mathrm{s}$. Moreover, the arm current $i_{z 1}$ and injected arm voltage $v_{q 1}$ were measured independently from the control system via digital sampling oscilloscope (LeCroy 24MXs-B) with current probe (LeCroy AP015) and voltage probe (Testec TT-SI 9110). A PZ4000 power analyzer was connected to one arm to measure the losses $P_{z 1}$ as depicted in Figure 1a and the control system was used to calculate the energy ripple $\Delta e_{z 1}$.

Measurements and nominal results of the low-voltage MMC test-bench for the set of optimal current references and cases A, B, and C are shown in Figure 8. As can be seen, experimental results depict the trade-off between the normalized energy ripple and losses, with a small error in each axis with respect to the nominal Pareto frontier. It is expected for the experimental losses to be slightly higher than the theoretical value, as the current ripple and switching losses are not included in the model. However, this deviation is approximately constant for the whole operating range, as the current ripple and switching losses depend on the switching frequency, which is constant and independent of the injected circulating current. Regardless of this deviation, it is experimentally verified that the total cell losses can be reduced with respect to zero circulating current, with a great reduction of the energy ripple. This reduction in both objectives with respect to case A for $\phi=0^{\circ}$ has been reported in [37,38], while the always negative effect of the second harmonic on the losses for $\phi=90^{\circ}$ is observed in [39]. Regarding the energy ripple model, the voltage drop of the semiconductors is an important source of error in low-voltage MMCs, which is not taken into account by the model. Hence, the energy ripple error is small for zero circulating current, and, as the circulating current is increased, the voltage drop and energy ripple error increase. Nevertheless, this distortion is even smaller in 
medium- and high-voltage MMCs, as the cell voltages are higher in comparison to the voltage drop of its semiconductors.

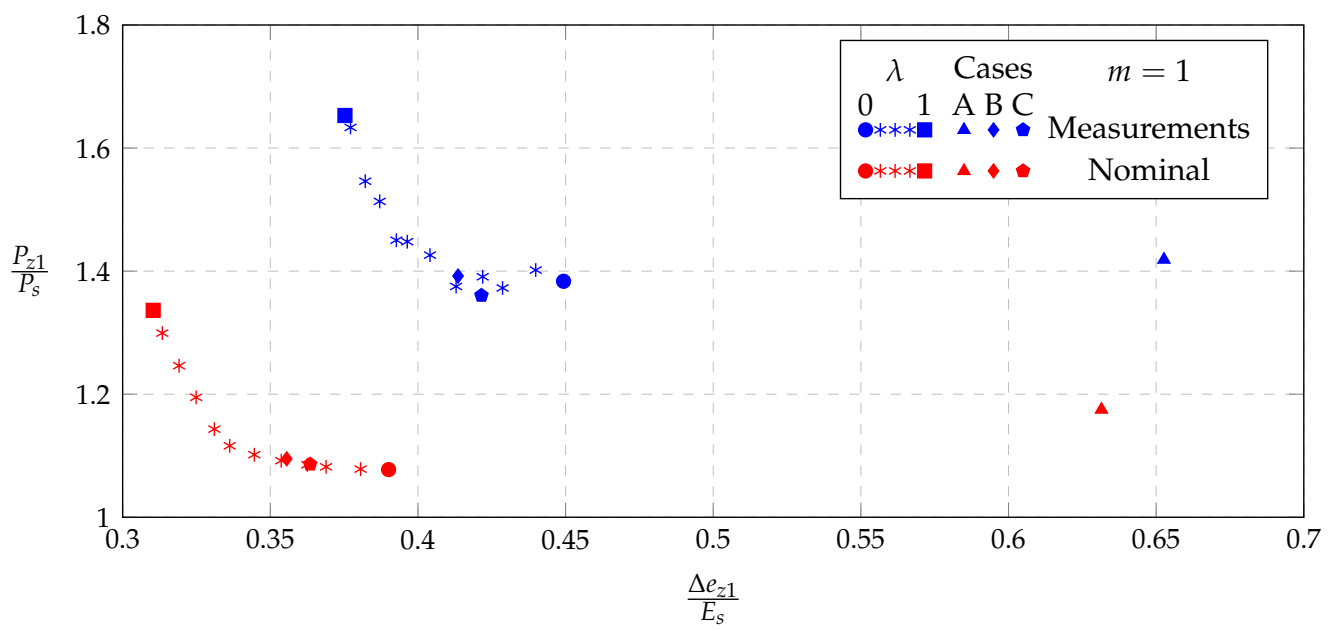

Figure 8. Measurement results and nominal values of the Pareto frontier and the cases $\mathrm{A}, \mathrm{B}$, and $\mathrm{C}$ for a low-voltage MMC with $R_{z}=166.9 \mathrm{~m} \Omega, V_{T z}=4.522 \mathrm{~V}, \hat{I}=10 \mathrm{~A}, \phi=0^{\circ}$ and $m=1$.

Figure 9 shows the arm energy, cell voltage, arm current, and injected arm voltage for the points given by $\lambda \in\{0,0.555,1\}$ on the measured Pareto frontier in Figure 8 . Nominal values are shown in red, measured values in blue. Figure 10 shows the same quantities for the cases A, B, and C.

The measured arm energy as well as the measured cell voltage exhibit lower minimum and higher maximum values than their nominal counterpart, leading to higher peak-topeak ripple in the experiment than predicted by the model. This error is mainly caused by the simplifying assumptions of a lossless system during the modeling, which is not rejected by the energy balancing controller. The mean values of the measured and nominal energies coincide much better because the stored energy $e_{s 0}$ is controlled by a dedicated PI controller, as depicted in the block diagram in Figure 7. The measured arm currents in Figures 9 and 10 match well to their nominal values, since all currents are controlled by their respective controller and the current components demanded by the energy balancing are very small in contrast to the nominal values. As visible from the bottom rows in Figures 9 and 10, the injected arm voltages exhibit distinctive switching patterns, especially when the number of active cells is high. This is caused by the higher control margin for the optimized regimes in comparison to case A in Figure 10. In all cases, the stored energy is controlled to be $e_{s 0}=45.39 \mathrm{~J}$, which is just enough for case A to operate with no control margin, as can be seen from the omitted pulses in $v_{q 1}$ at $t \approx 11 \mathrm{~ms}$. The optimized cases have higher control margin and, consequently, no pulses are omitted. 

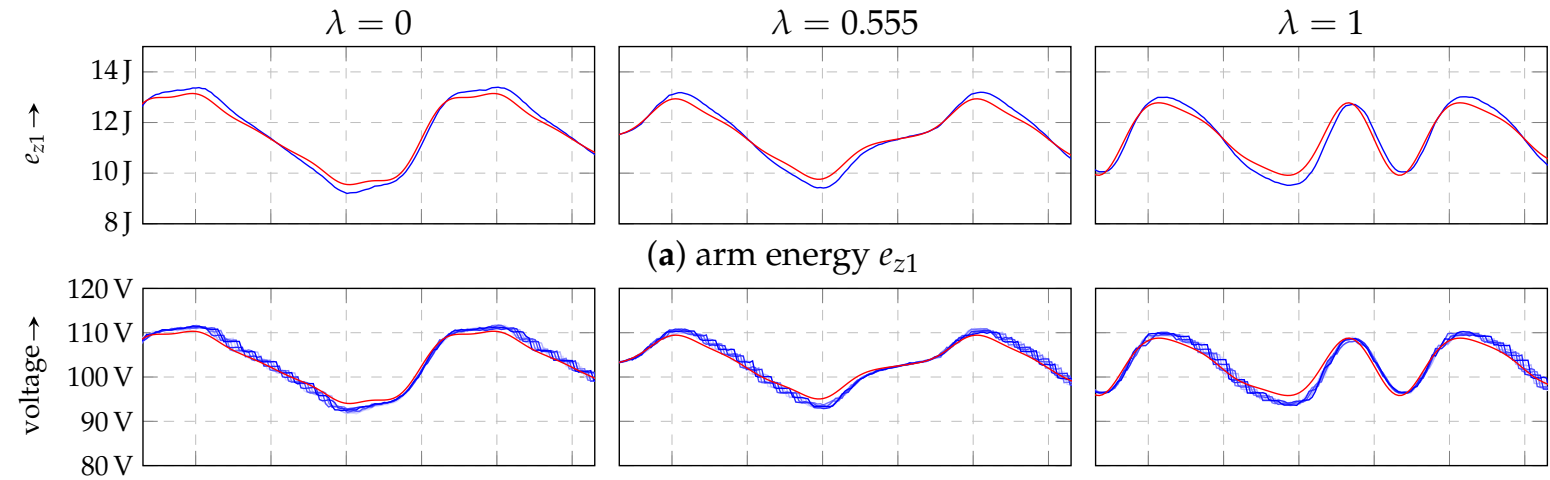

(a) arm energy $e_{z 1}$

(b) nominal equivalent cell voltage divided by $n$ (red), measured cell voltages (blue)
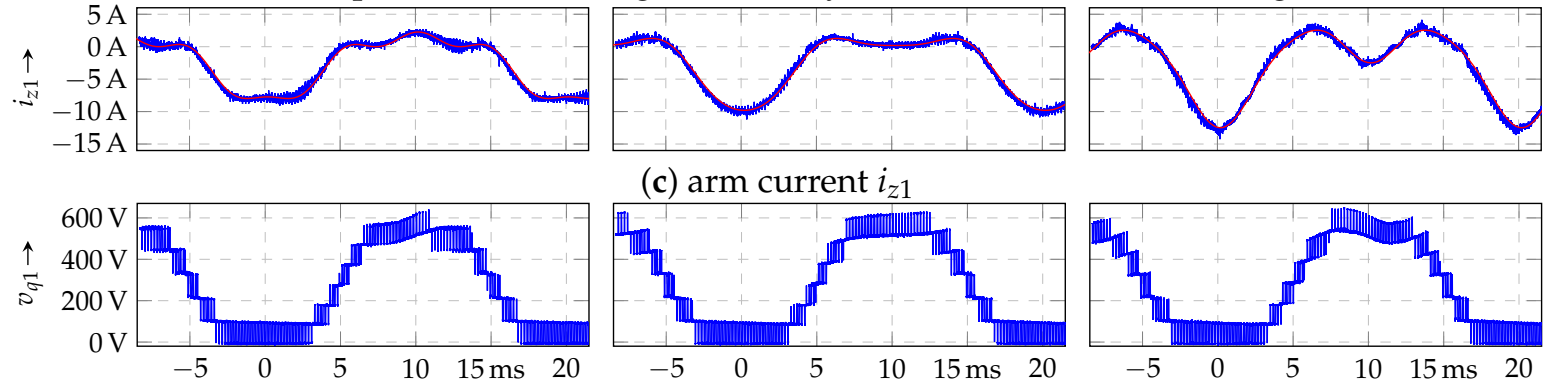

(c) arm current $i_{z 1}$
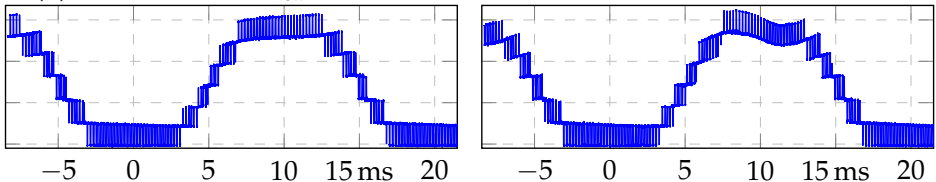

(d) arm voltage $v_{q 1}$

Figure 9. Comparison of Pareto optimal solutions with $\lambda \in\{0,0.555,1\}$ for a low-voltage MMC with $R_{z}=166.9 \mathrm{~m} \Omega$, $V_{T z}=4.522 \mathrm{~V}, \hat{I}=10 \mathrm{~A}, \phi=0^{\circ}$, and $m=1$ : (a) Arm energy $e_{z 1}$, (b) cell voltages, and scaled nominal equivalent cell voltage, (c) arm current $i_{z 1}$, (d) and injected arm voltage $v_{q 1}$. Nominal values are shown in red, measured values in blue. Measured (c) current and (d) voltage via oscilloscope (LeCroy 24MXs-B), using high bandwidth current probe (LeCroy AP015) and voltage probe (Testec TT-SI 9110).

A
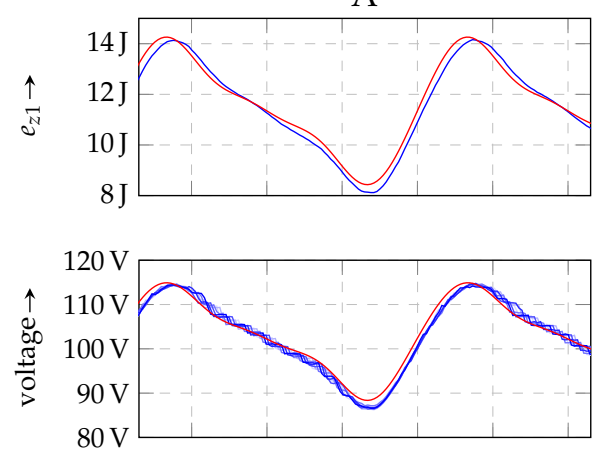

(b) nominal equivalent cell voltage divided by $n$ (red), measured cell voltages (blue)

C

(a) arm energy $e_{z 1}$

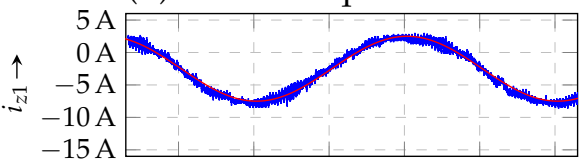

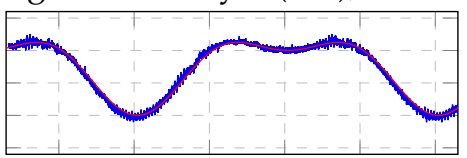

(c) arm current $i_{z 1}$

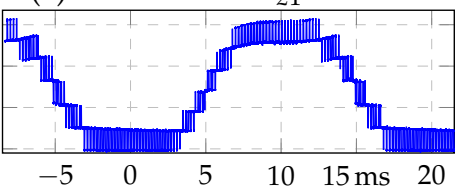

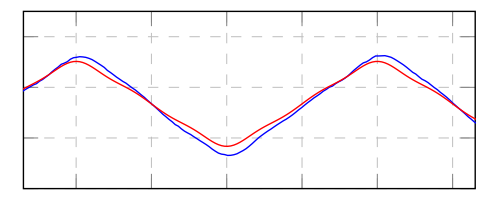
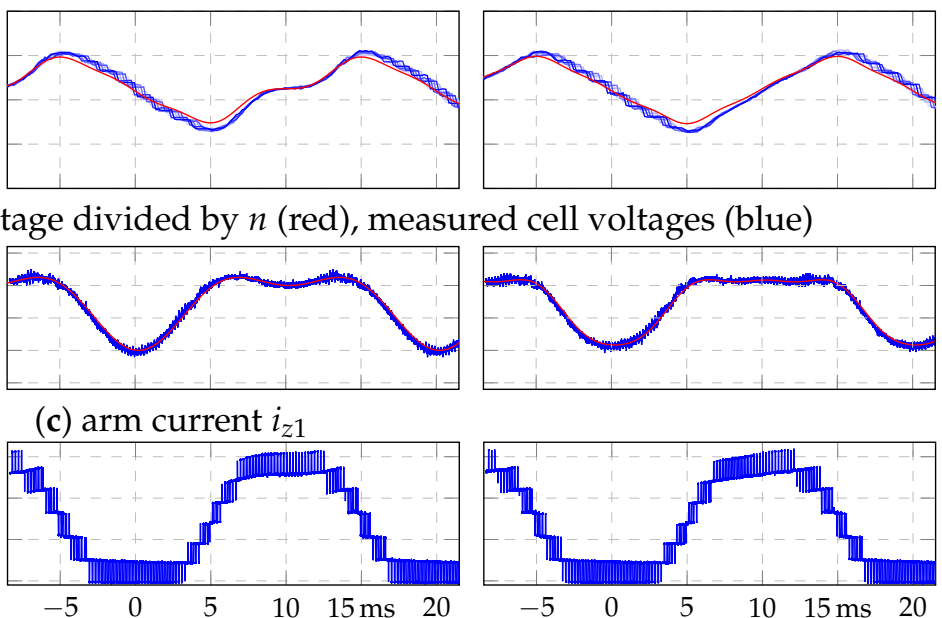

(d) arm voltage $v_{q 1}$

Figure 10. Comparison of Cases A, B, and C from [28] for a low-voltage MMC with $R_{z}=166.9 \mathrm{~m} \Omega, V_{T z}=4.522 \mathrm{~V}, \hat{I}=10 \mathrm{~A}$, $\phi=0^{\circ}$, and $m=1$ : (a) Arm energy $e_{z 1}$, (b) cell voltage, and scaled nominal equivalent cell voltage, (c) arm current $i_{z 1}$, and (d) injected arm voltage $v_{q 1}$. Nominal values are shown in red, measured values in blue. Measured (c) current and (d) voltage via oscilloscope (LeCroy 24MXs-B), using high bandwidth current probe (LeCroy AP015) and voltage probe (Testec TT-SI 9110). 


\section{Conclusions}

A method to numerically solve the multi-objective problem of minimizing the arm power losses and energy ripple of an MMC is proposed. For symmetric operation, the algorithm utilizes the 2nd and 4th harmonics because higher order harmonics may lie beyond the bandwidth of the current controller, while their contribution is damped by the cell capacitance. Thanks to the energy based analysis, the arm stored energy is not fixed or constrained by the algorithm, allowing the stored energy to be set independently of the optimal solution. The optimization process includes the calculation of the current solutions, which allows for a fast calculation of the objective function and its gradient, improving the convergence and computation speed of the minimization algorithm. Moreover, symmetries in the Pareto frontier reduce the search space, which further improve the computation speed.

Experimental results reveal the competitive nature of the energy ripple and power losses, in which the best compromise in the set of optimal solutions can be chosen depending on the application requirements. Furthermore, measurements show that the losses and energy ripple can be simultaneously reduced with respect to zero circulating current injection. Small deviations in the calculation of the power losses and energy ripple are identified, explained by the additional switching and ripple losses, and the semiconductor voltage drop. However, in medium- and high-voltage MMCs, these deviations are even smaller, as the number of cells is higher leading to a lower current ripple, while the cell voltage is higher in relation to the semiconductor voltage drop.

Future work could extend the optimization by giving up the fixed relationship between common-mode voltage and $\mathrm{AC}$ voltage, in order to improve the results especially for low modulation index operation. Moreover, the losses can be associated with the respective volume of the heat sink and the energy ripple can be linked to the volume of the cell capacitor to obtain an optimal combination of both in order to minimize the volume of the MMC.

Author Contributions: Conceptualization A.G., M.L., H.F. and M.A.P.; software, M.L. and H.F.; prototype test-bench experiments, H.F. and M.L.; writing—original draft preparation, H.F. and M.L.; writing-review and editing, A.G. and M.A.P.; visualization, H.F. and M.L. All authors have read and agreed to the published version of the manuscript.

Funding: This research was funded by Deutsche Forschungsgemeinschaft, DFG, grant GE 2502/5-1, by Fondecyt Project 1181839, by ANID MEC 80180050, by ANID-Basal Project FB0008 and by SERC (ANID/FONDAP/15110019).

Institutional Review Board Statement: Not applicable.

Informed Consent Statement: Not applicable.

Acknowledgments: We would like to acknowledge the support given by Technische Universität Dresden and Universidad Técnica Federico Santa María.

Conflicts of Interest: The authors declare no conflict of interest.

Suppliers: MATLAB software version 9.7.0.1190202 (R2019b): The MathWorks, Inc. Natick, Massachusetts (USA); Digital sampling oscilloscope and current probe: Teledyne Technologies Inc. Thousand Oaks, California (USA); voltage probes: TESTEC Elektronik GmbH, Frankfurt, Germany; grid emulator: Control Intel-ligent de l'Energia, coop Barcelona, Spain; rapid control prototyping system: dSPACE GmbH Paderborn, Germany.

\section{References}

1. Lesnicar, A.; Marquardt, R. An Innovative Modular Multilevel Converter Topology Suitable for a Wide Power Range. In Proceedings of the Power Tech Conference, Bologna, Italy, 23-26 June 2003; Volume 3.

2. Oates, C. A Methodology for Developing 'Chainlink' Converters. In Proceedings of the 13th European Conference on Power Electronics and Applications, EPE, Barcelona, Spain, 8-10 September 2009.

3. Akagi, H. Classification, Terminology, and Application of the Modular Multilevel Cascade Converter (MMCC). IEEE Trans. Power Electron. 2011, 26, 3119-3130. [CrossRef] 
4. Martinez-Rodrigo, F.; Ramirez, D.; Rey-Boue, A.B.; De Pablo, S.; Herrero-de Lucas, L.C. Modular multilevel converters: Control and applications. Energies 2017, 10, 1709. [CrossRef]

5. Hiller, M.; Krug, D.; Sommer, R.; Rohner, S. A New Highly Modular Medium Voltage Converter Topology for Industrial Drive Applications. In Proceedings of the 13th European Conference on Power Electronics and Applications, EPE, Barcelona, Spain, 8-10 September 2009.

6. Rohner, S.; Weber, J.; Bernet, S. Continuous model of Modular Multilevel Converter with experimental verification. In Proceedings of the IEEE Energy Conversion Congress and Exposition, ECCE, Phoenix, AZ, USA, 17-22 September 2011; pp. 4021-4028.

7. Marquardt, R. Modular Multilevel Converter: An universal concept for HVDC-Networks and extended DC-Bus-applications. In Proceedings of the International Power Electronics Conference, IPEC, Sapporo, Japan, 21-24 June 2010; pp. 502-507.

8. Himmelmann, P.; Hiller, M.; Krug, D.; Beuermann, M. A new Modular Multilevel Converter for Medium Voltage High Power Oil \& Gas Motor Drive Applications. In Proceedings of the 18th European Conference on Power Electronics and Applications EPE'16 ECCE Europe, Karlsruhe, Germany, 5-9 September 2016.

9. Acharya, A.B.; Ricco, M.; Sera, D.; Teodorescu, R.; Norum, L.E. Arm power control of the modular multilevel converter in photovoltaic applications. Energies 2019, 12, 1620. [CrossRef]

10. Acharya, A.B.; Sera, D.; Teodorescu, R.; Norum, L.E. Modular Multilevel Converter for Photovoltaic Application with High Energy Yield under Uneven Irradiance. Energies 2020, 13, 2619. [CrossRef]

11. Dekka, A.; Wu, B.; Fuentes, R.L.; Perez, M.; Zargari, N.R. Evolution of Topologies, Modeling, Control Schemes, and Applications of Modular Multilevel Converters. IEEE J. Emerg. Sel. Top. Power Electron. 2017, 5, 1631-1656. [CrossRef]

12. Liu, M.; Li, Z.; Yang, X. A universal mathematical model of modular multilevel converter with half-bridge. Energies 2020, $13,4464$. [CrossRef]

13. Huang, L.; Yang, X.; Xu, P.; Zhang, F.; Ma, X.; Liu, T.; Hao, X.; Liu, W. The Evolution and Variation of Sub-Module Topologies with DC-fault Current Clearing Capability in MMC-HVDC. In Proceedings of the IEEE 3rd International Future Energy Electronics Conference and ECCE Asia IFEEC 2017-ECCE Asia, Kaohsiung, Taiwan, 3-7 June 2017; pp. 1938-1943.

14. Xu, Z.; Xiao, H.; Zhang, Z. Selection methods of main circuit parameters for modular multilevel converters. IET Renew. Power Gener. 2016, 10, 788-797. [CrossRef]

15. Oates, C. Modular Multilevel Converter Design for VSC HVDC Applications. IEEE J. Emerg. Sel. Top. Power Electron. 2015, 3, 505-515. [CrossRef]

16. Moranchel, M.; Bueno, E.; Sanz, I.; Rodríguez, F.J. New approaches to circulating current controllers for modular multilevel converters. Energies 2017, 10, 86. [CrossRef]

17. Perez, M.A.; Bernet, S.; Rodriguez, J.; Kouro, S.; Lizana, R. Circuit Topologies, Modeling, Control Schemes, and Applications of Modular Multilevel Converters. IEEE Trans. Power Electron. 2015, 30, 4-17. [CrossRef]

18. Engel, S.P.; De Doncker, R.W. Control of the Modular Multi-Level Converter for minimized cell capacitance. In Proceedings of the EPE'11ECCE, Birmingham, UK, 30 August-1 September 2011.

19. Picas, R.; Pou, J.; Ceballos, S.; Zaragoza, J.; Konstantinou, G.; Agelidis, V.G. Optimal Injection of Harmonics in Circulating Currents of Modular Multilevel Converters for Capacitor Voltage Ripple Minimization. In Proceedings of the IEEE ECCE Asia Downunder, Melbourne, Australia, 3-6 June 2013; pp. 318-324.

20. Korn, A.J.; Winkelnkemper, M.; Steimer, P. Low Output Frequency Operation of the Modular Multi-Level Converter. In Proceedings of the IEEE Energy Conversion Congress and Exposition ECCE, Atlanta, GA, USA, 12-16 September 2010; pp. $3993-3997$.

21. He, L.; Zhang, K.; Xiong, J.; Fan, S.; Xue, Y. Low-Frequency Ripple Suppression for Medium-Voltage Drives Using Modular Multilevel Converter With Full-Bridge Submodules. IEEE J. Emerg. Sel. Top. Power Electron. 2016, 4, 657-667. [CrossRef]

22. Fuchs, S.; Jeong, M.; Biela, J. Reducing the Energy Storage Requirements of Modular Multilevel Converters with Optimal Capacitor Voltage Trajectory Shaping. In Proceedings of the EPE'20 ECCE Europe, Online Conference, 7-11 September 2020.

23. Townsend, D.; Mirzaeva, G.; Goodwin, G.C. Capacitance Minimization in Modular Multilevel Converters: A Reliable and Computationally Efficient Algorithm to Identify Optimal Circulating Currents and Zero-Sequence Voltages. In Proceedings of the IEEE 12th International Conference on Power Electronics and Drive Systems PEDS, Honolulu, HI, USA, 12-15 December 2017; pp. 98-104.

24. Yang, L.; Li, Y.; Li, Z.; Wang, P.; Xu, S.; Gou, R. Loss Optimization of MMC by Second-Order Harmonic Circulating Current Injection. IEEE Trans. Power Electron. 2018, 33, 5739-5753. [CrossRef]

25. Wang, J.; Han, X.; Ma, H.; Bai, Z. Analysis and Injection Control of Circulating Current for Modular Multilevel Converters. IEEE Trans. Ind. Electron. 2019, 66, 2280-2290. [CrossRef]

26. Pou, J.; Ceballos, S.; Konstantinou, G.; Agelidis, V.G.; Picas, R.; Zaragoza, J. Circulating Current Injection Methods Based on Instantaneous Information for the Modular Multilevel Converter. IEEE Trans. Ind. Electron. 2015, 62, 777-788. [CrossRef]

27. Zhao, C.; Li, Y.; Li, Z.; Wang, P.; Ma, X.; Luo, Y. Optimized Design of Full-Bridge Modular Multilevel Converter With Low Energy Storage Requirements for HVdc Transmission System. IEEE Trans. Power Electron. 2018, 33, 97-109. [CrossRef]

28. Fehr, H.; Gensior, A. Model-Based Circulating Current References for MMC Cell Voltage Ripple Reduction and Loss-Equivalent Arm Current Assessment. In Proceedings of the EPE'19 ECCE Europe, Genova, Italy, 3-5 September 2019.

29. Bärnklau, H.; Gensior, A.; Bernet, S. Derivation of an Equivalent Submodule per Arm for Modular Multilevel Converters. In Proceedings of the EPE-PEMC ECCE Europe, Novi Sad, Serbia, 4-6 September 2012; pp. LS2a.2-1-LS2a.2-5. 
30. Fehr, H.; Gensior, A. Eigenvalue Optimization of the Energy Balancing Feedback for Modular Multilevel Converters. IEEE Trans. Power Electron. 2019, 34, 11482-11495. [CrossRef]

31. Antonopoulos, A.; Ängquist, L.; Harnefors, L.; Nee, H.P. Optimal Selection of the Average Capacitor Voltage for Variable-Speed Drives With Modular Multilevel Converters. IEEE Trans. Power Electron. 2015, 30, 227-234. [CrossRef]

32. Shinoda, K.; Benchaib, A.; Dai, J.; Guillaud, X. Analysis of the Lower Limit of Allowable Energy in Modular Multilevel Converters. In Proceedings of the EPE'18 ECCE Europe, Riga, Latvia, 17-21 September 2018.

33. Jones, P.S.; Davidson, C.C. Calculation of Power Losses for MMC-based VSC HVDC Stations. In Proceedings of the 15th European Conference on Power Electronics and Applications EPE, Lille, France, 2-6 September 2013.

34. Murata, T.; Ishibuchi, H.; Tanaka, H. Multi-objective genetic algorithm and its applications to flowshop scheduling. Comput. Ind. Eng. 1996, 30, 957-968. [CrossRef]

35. Weisstein, E.W. Multiple-Angle Formulas. From MathWorld-A Wolfram Web Resource. Available online: https://mathworld.wo lfram.com/Multiple-AngleFormulas.html (accessed on 24 April 2020).

36. Lopez, M.; Fehr, H. Matlab Scripts for MMC Pareto Optimization; Technische Universität Dresden: Dresden, Germany, 2020. [CrossRef]

37. Marchesoni, M.; Vaccaro, L. Study of the MMC circulating current for optimal operation mode in HVDC applications. In Proceedings of the EPE'15 ECCE-Europe, Geneva, Switzerland, 8-10 September 2015.

38. Dudin, A.; Fidelak, A.; Petzoldt, J. Effect of the loss-minimizing circulating current on the Modular Multilevel Converter with IGBT switches. In Proceedings of the EPE'16 ECCE Europe, Karlsruhe, Germany, 5-9 September 2016.

39. Bordignon, P.; Maragliano, G.; Marchesoni, M.; Vaccaro, L. Effects of the 2nd Order Harmonic Leg Current in a MMC converter. In Proceedings of the EPE'14-ECCE Europe, Lappeeranta, Finland, 26-28 August 2014. 